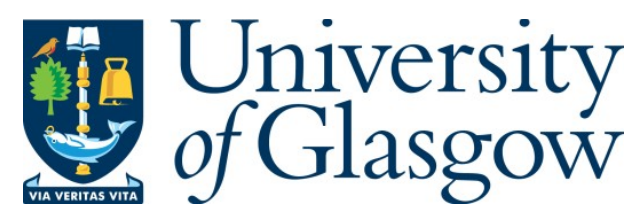

Li, K., Ogden, R. W., and Holzapfel, G. A. (2016) Computational method for excluding fibers under compression in modeling soft fibrous solids. European Journal of Mechanics A/Solids, 57, pp. 178-193.

There may be differences between this version and the published version. You are advised to consult the publisher's version if you wish to cite from it.

http://eprints.gla.ac.uk/119640/

Deposited on: 26 May 2016

Enlighten - Research publications by members of the University of Glasgow http://eprints.gla.ac.uk 


\title{
Computational Method for Excluding Fibers Under Compression in Modeling Soft Fibrous Solids
}

\author{
Kewei $\mathrm{Li}^{1}$, Ray W. Ogden ${ }^{2}$ and Gerhard A. Holzapfel ${ }^{1, \dagger}$ \\ ${ }^{1}$ Institute of Biomechanics, Graz University of Technology \\ Stremayrgasse 16-II, 8010 Graz, Austria \\ ${ }^{2}$ School of Mathematics and Statistics, University of Glasgow \\ University Gardens, Glasgow G12 8QW, Scotland, UK
}

Nov 6, 2015

\begin{abstract}
Soft fibrous solids often consist of a matrix reinforced by fibers that render the material anisotropic. Recently a fiber dispersion model was proposed on the basis of a weighted strain-energy function using an angular integration approach for both planar and three-dimensional fiber dispersions (G.A. Holzapfel and R.W. Ogden: Eur. J. Mech. A/Solids, 49 (2015) 561569). This model allows the exclusion of fibers under compression. In the present study computational aspects of the model are documented. In particular, we provide expressions for the elasticity tensor and the integration boundary that admits only fibers which are extended. In addition, we give a brief description of the finite element implementation for both $2 \mathrm{D}$ and $3 \mathrm{D}$ models which make use of the von Mises distribution to describe the dispersion of the fibers. The performance and the finite element implementations of the 2D and 3D fiber dispersion models are illustrated by means of uniaxial extension in the mean fiber direction and more general directions, and simple shear with different mean fiber directions. The finite element results are in perfect agreement with the solutions computed from analytical formulas.
\end{abstract}

Keywords: Constitutive modeling; soft fibrous solids; finite element method, fiber dispersion; anisotropy; exclusion of compressed fibers

\section{Introduction}

In many soft fibrous solids, including biological tissues, there exists a matrix reinforced by embedded fibers which, in general, induce anisotropy in the material. For some materials the matrix can be treated as homogeneous and isotropic. The fibers may be distributed within the

\footnotetext{
${ }^{\dagger}$ To whom correspondence should be addressed. Email address: holzapfel@tugraz . at
} 
matrix in various ways. Specifically, in human arterial walls the collagen fibers are not perfectly aligned but are dispersed around a mean direction. Such a fiber dispersion has been observed in, for example, human arterial walls [1-5], the myocardium [6, 7], corneas [8, 9] and articular cartilage [10]. In particular, recent extensive experimental results [4] have shown that the collagen fiber dispersion in each of the layers of (healthy) human thoracic and abdominal aortas and iliac arteries is non-symmetric, in contrast to the rotationally symmetric fiber dispersion assumed in previous studies; see, for example, [11]. In order to improve understanding of the mechanical properties of such tissues, constitutive modeling is essential.

Motivated by the specific structural arrangements of collagen fibers, various constitutive relations have been developed. Fiber dispersion has been represented in such constitutive relations either by direct incorporation in a strain-energy function via a probability density function (PDF) or by a generalized structure tensor. Following [12] these two approaches are referred to as 'angular integration' (AI) and 'generalized structure tensor' (GST), respectively. For a short survey of the main existing constitutive models that account for dispersion of collagen fibers by using either the AI approach (due to Lanir [13]) or the GST approach, as reviewed in [14]. In particular, our group has developed a constitutive relation for the modeling of arterial layers with a rotationally symmetric fiber dispersion [11]. Recently, this model has been extended to a more general case [14] for which a non-symmetric fiber dispersion can also be captured.

Generally, the role of the fibers is primarily mechanical, providing the material with increased stiffness and strength. The fibers are elongated when loaded in tension, and it is often assumed that they do not contribute to the overall mechanical response of the material in compression. The computational implementation of this assumption requires a tension-compression 'switch' which eliminates the mechanical contribution of each fiber that is in compression. However, as pointed out in [15], such a condition has not been interpreted correctly in the literature and in finite element programs; see, for example, [16].

A Heaviside step function is sometimes introduced to eliminate the mechanical influence of the compressed fibers; see [17-20]. Theoretically, this method could successfully exclude the contribution of the compressed fibers from the total strain-energy function. However, as indicated in [19], the presence of the Heaviside function renders the stress and elasticity tensors discontinuous. In the recent paper [15] we have proposed a modified fiber dispersion model which incorporates a weighted strain-energy function that allows the exclusion of fibers under compression without the need for a Heaviside function. This model, which is based on the AI approach, was developed for planar and three-dimensional fiber dispersions and enables the stress and the elasticity tensors to be calculated in a straightforward way. However, the 
computational aspects of this modified model, specifically the form of the elasticity tensor and the integration boundary that admits only fibers which are extended, are not yet documented. Therefore, the aim of this study is to further develop this model for the purpose of computational implementation.

The present study is structured as follows. In Section 2 we present the continuum mechanical framework for the modified fiber dispersion model in a decoupled form suitable for finite element implementation, including the Cauchy stress and the elasticity tensors for both planar and three-dimensional fiber distributions. The boundary of the integration domain is also discussed for different deformation states. In Section 3 we introduce an adaptive finite element integration scheme for the numerical integration required for the stress and the elasticity tensors in the appropriate domain. In Section 4 the theory introduced in Section 2 is applied to several examples using the finite element scheme from Section 3. In particular, six representative numerical simulations are presented with the aim of demonstrating the efficacy of the proposed computational method. Finally, Section 5 summarizes the developed method and discusses possible future developments of the present study.

\section{Continuum Mechanical Framework}

In this section we outline the basic notation and fundamental results of nonlinear continuum mechanics in order to establish the mathematical description of fiber dispersion models, including the corresponding Cauchy stress and elasticity tensors. In particular, the integration boundary in the deformation space within which fibers are extended is also introduced.

\subsection{Kinematics}

Let $\mathcal{B}_{0}$ be a (stress-free) reference configuration of a continuum body and $\mathcal{B}$ its deformed configuration. The deformation map $\chi(\mathbf{X})$ transforms a material point $\mathbf{X} \in \mathcal{B}_{0}$ into a spatial point $\mathbf{x} \in \mathcal{B}$. With this deformation map we define the deformation gradient $\mathbf{F}(\mathbf{X})=\partial \boldsymbol{\chi}(\mathbf{X}) / \partial \mathbf{X}$ and its determinant $J=\operatorname{det} \mathbf{F}(\mathbf{X})$, where $J$ is the local volume ratio; we require $J>0$.

Following the multiplicative decomposition of the deformation gradient in [21, 22] we decouple $\mathbf{F}$ into a spherical (dilatational) part $J^{1 / 3} \mathbf{I}$ and a unimodular (distortional) part $\overline{\mathbf{F}}=$ $J^{-1 / 3} \mathbf{F}$, with $\operatorname{det} \overline{\mathbf{F}} \equiv 1$. We define the right Cauchy-Green tensor $\mathbf{C}=\mathbf{F}^{\mathrm{T}} \mathbf{F}$ and its modified counterpart $\overline{\mathbf{C}}=\overline{\mathbf{F}}^{\mathrm{T}} \overline{\mathbf{F}}$, respectively, with the related invariants $I_{1}=\operatorname{tr} \mathbf{C}$ and $\bar{I}_{1}=\operatorname{tr} \overline{\mathbf{C}}$. 


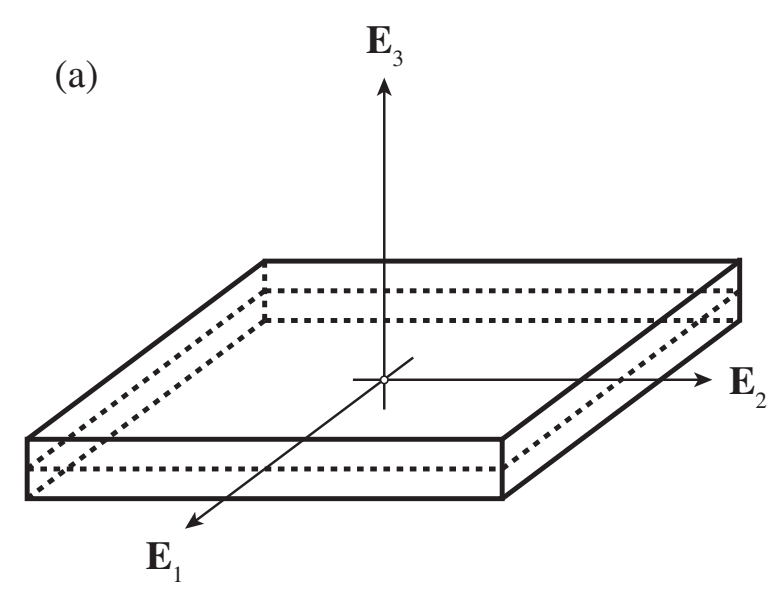

(b)

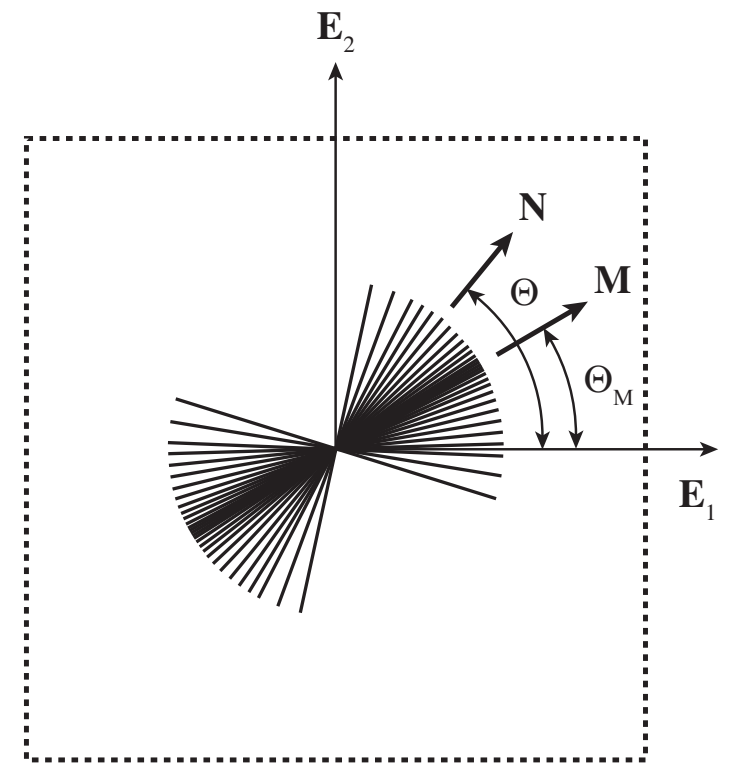

Figure 1: (a) A fibrous sheet with uniform thickness whose major surfaces are normal to the direction $\mathbf{E}_{3}$; (b) a planar fiber dispersion described by a unit vector $\mathbf{N}$ representing an arbitrary fiber direction normal to $\mathbf{E}_{3}$ defined by the angle $\Theta$ with respect to $\mathbf{E}_{1}$. The mean fiber direction $\mathbf{M}$ makes an angle $\Theta_{\mathrm{M}}$ with $\mathbf{E}_{1}$.

\subsection{Planar Fiber Dispersion Model}

The modified fiber dispersion model [15] that accounts only for fibers under extension requires numerical integration in the sub-domain of a unit sphere for which the fiber stretch is greater than one. For some soft biological tissues such as arterial walls the fiber dispersion in the thickness direction is smaller than in the in-plane direction [4], and for our present purposes we neglect the out-of-plane dispersion. We treat the material as incompressible, elastic and fiber-reinforced with a locally planar fiber dispersion. Without loss of generality we choose the thickness direction in such a material as the $\mathbf{E}_{3}$ Cartesian axis. Hence, an arbitrary in-plane fiber direction within a dispersion about a mean fiber direction $\mathbf{M}$ may be described by a unit vector $\mathbf{N}$ in the reference configuration as

$$
\mathbf{N}(\Theta)=\cos \Theta \mathbf{E}_{1}+\sin \Theta \mathbf{E}_{2},
$$

where $\mathbf{E}_{1}$ and $\mathbf{E}_{2}$ are the in-plane unit rectangular Cartesian basis vectors, and $\Theta$ is the angle between the fiber direction $\mathbf{N}$ and $\mathbf{E}_{1}$, as shown in Figure 1. Also shown in Figure 1 is the mean fiber direction $\mathbf{M}$ and the angle $\Theta_{\mathrm{M}}$ that it makes with the $\mathbf{E}_{1}$ direction. Analogously to (1) we 
may write

$$
\mathbf{M}=\cos \Theta_{\mathrm{M}} \mathbf{E}_{1}+\sin \Theta_{\mathrm{M}} \mathbf{E}_{2}
$$

in the reference configuration, where $\Theta_{\mathrm{M}}$ is a constant.

Since we are considering elastic materials, we assume that there exists a strain-energy function $\Psi(\mathbf{C},\{\mathbf{N}\})$, where $\{\mathbf{N}\}$ implies the dependence on the distribution of $\mathbf{N}$, that depends on the macroscopic deformation through $\mathbf{C}$, the underlying material structure through each direction $\mathbf{N}$, and a PDF $\rho(\Theta)$ that describes the fiber alignment and dispersion. For computational purposes, we assume that the strain-energy function can be decoupled as [23]

$$
\Psi(\mathbf{C},\{\mathbf{N}\})=\Psi_{\text {vol }}(J)+\Psi_{\text {iso }}(\overline{\mathbf{C}},\{\mathbf{N}\}),
$$

where the function $\Psi_{\text {vol }}$ is a purely volumetric contribution while $\Psi_{\text {iso }}$ represents the energy contribution of an isochoric (volume preserving) deformation through $\overline{\mathbf{C}}$. Suppose now the total isochoric strain-energy function $\Psi_{\text {iso }}$ is the superposition of the energies contributed by the (non-collagenous) ground matrix and the collagen fibers, i.e. [24]

$$
\Psi_{\text {iso }}=\Psi_{\mathrm{g}}(\overline{\mathbf{C}})+\Psi_{\mathrm{f}}(\overline{\mathbf{C}},\{\mathbf{N}\}) .
$$

Following $[15,24]$ we model the ground matrix as a neo-Hookean material $\Psi_{\mathrm{g}}\left(\bar{I}_{1}\right)=\mu\left(\bar{I}_{1}-\right.$ $3) / 2$, where the parameter $\mu$ is the shear modulus in the reference configuration. The isochoric strain energy contributed by the fibers per unit reference volume associated with the direction $\mathbf{N}$ is assumed to be a function of the fiber stretch only. Thus, we adopt a modified form of the standard fiber reinforcing model [25] for the contribution of a fiber along $\mathbf{N}$ in which $\bar{I}_{4}$ is used instead of $I_{4}$. This is given by

$$
\Psi_{\mathrm{n}}\left(\bar{I}_{4}(\mathbf{N})\right)=\frac{\nu}{2}\left(\bar{I}_{4}(\mathbf{N})-1\right)^{2},
$$

where $\nu$ is a non-negative material constant with the dimension of stress and the modified fourth invariant is $\bar{I}_{4}(\mathbf{N})=\overline{\mathbf{C}}: \mathbf{N} \otimes \mathbf{N}=J^{-2 / 3} I_{4}(\mathbf{N})$, with $I_{4}(\mathbf{N})=\mathbf{C}: \mathbf{N} \otimes \mathbf{N}$. Note here that $\nu$ is the stiffness of the fiber in the direction of $\mathbf{N}$. The invariant $I_{4}(\mathbf{N})$ is the square of the fiber stretch in the direction $\mathbf{N}$; see, for example, [23]. Note that (5) is entirely appropriate to describe the behavior of single collagen fibers, as exemplified in [26]. Now we write the isochoric part of the strain-energy function of the fiber dispersion, per unit reference area of the considered plane, weighted with $\rho(\Theta)$ as

$$
\Psi_{\mathrm{f}}=\frac{1}{\pi} \int_{\Sigma} \rho(\Theta) \Psi_{\mathrm{n}}\left(\bar{I}_{4}(\Theta)\right) \mathrm{d} \Theta,
$$

where $\Sigma \in\left\{\Theta \in[-\pi / 2, \pi / 2] \mid I_{4}(\Theta)>1\right\}$ defines the region in which fibers are extended, and $\Theta$ is the angle of an arbitrary fiber orientation $\mathbf{N}$, as shown in Figure 1(b). Note that 
we are restricting attention to the half circle $\Theta \in[-\pi / 2, \pi / 2]$ since $\mathbf{N}$ and $-\mathbf{N}$ represent the same 'fiber'. The PDF $\rho(\Theta)$ represents the probability density of fibers at orientation $\mathbf{N}$ in the reference configuration. It satisfies the normalization condition

$$
\frac{1}{\pi} \int_{-\pi / 2}^{\pi / 2} \rho(\Theta) \mathrm{d} \Theta=1
$$

The total isochoric strain-energy function $\Psi_{\text {iso }}$ of the material now reads

$$
\Psi_{\text {iso }}=\Psi_{\mathrm{g}}\left(\bar{I}_{1}\right)+\frac{1}{\pi} \int_{\Sigma} \rho(\Theta) \Psi_{\mathrm{n}}\left(\bar{I}_{4}(\Theta)\right) \mathrm{d} \Theta .
$$

For fibrous solids with two or more fiber families, the strain energy of additional fiber families can be included additively in (8) in a similar way. Here we confine attention to materials reinforced with one family of dispersed fibers.

Note that the strain-energy function $\Psi_{\mathrm{f}}$ in (6) depends not only on $\overline{\mathbf{C}}$ and $\{\mathbf{N}\}$ but also on the fiber dispersion parameters which are included in $\rho(\Theta)$.

\subsubsection{Stress Tensors}

The second Piola-Kirchhoff stress tensor is given by $\mathbf{S}=2 \partial \Psi / \partial \mathbf{C}$. With the decoupled form of $\Psi$ we can identify two stress contributions: $\mathbf{S}=\mathbf{S}_{\mathrm{vol}}+\mathbf{S}_{\mathrm{iso}}$. The volumetric contribution $\mathbf{S}_{\mathrm{vol}}$ for the proposed model has been well documented; see [23]. Thus, here we focus only on the isochoric part, i.e.

$$
\mathbf{S}_{\text {iso }}=J^{-2 / 3} \operatorname{Dev} \overline{\mathbf{S}}, \quad \overline{\mathbf{S}}=2 \frac{\partial \Psi_{\text {iso }}}{\partial \overline{\mathbf{C}}},
$$

where $\overline{\mathbf{S}}$ denotes the so-called fictitious isochoric second Piola-Kirchhoff stress tensor [23]. The deviator in the Lagrangian configuration is defined by $\operatorname{Dev}(\bullet)=\mathbb{P}:(\bullet)$, where $\mathbb{P}=\mathbb{I}-\frac{1}{3} \mathbf{C}^{-1} \otimes$ $\mathbf{C}$ is a projection tensor that furnishes the correct deviatoric operator in the Lagrangian setting, and $(\mathbb{I})_{A B C D}=\frac{1}{2}\left(\delta_{A C} \delta_{B D}+\delta_{A D} \delta_{B C}\right)$ is the symmetric Lagrangian fourth-order identity tensor. With the specific isochoric strain-energy function (8), we obtain from $(9)_{2}$ that

$$
\overline{\mathbf{S}}=2 \psi_{\mathrm{g}}^{\prime}\left(\bar{I}_{1}\right) \mathbf{I}+\frac{2}{\pi} \int_{\Sigma} \rho(\Theta) \psi_{\mathrm{n}}^{\prime}\left(\bar{I}_{4}(\Theta)\right) \mathbf{N} \otimes \mathbf{N} \mathrm{d} \Theta,
$$

where $\mathbf{I}$ is the second-order identity tensor and $\psi_{\mathrm{g}}^{\prime}\left(\bar{I}_{1}\right)=\partial \Psi_{\mathrm{g}}\left(\bar{I}_{1}\right) / \partial \bar{I}_{1}, \psi_{\mathrm{n}}^{\prime}=\partial \Psi_{\mathrm{n}}\left(\bar{I}_{4}\right) / \partial \bar{I}_{4}$, and, from (1),

$$
\mathbf{N} \otimes \mathbf{N}=\cos ^{2} \Theta \mathbf{E}_{1} \otimes \mathbf{E}_{1}+\sin ^{2} \Theta \mathbf{E}_{2} \otimes \mathbf{E}_{2}+\sin \Theta \cos \Theta\left(\mathbf{E}_{1} \otimes \mathbf{E}_{2}+\mathbf{E}_{2} \otimes \mathbf{E}_{1}\right) .
$$


Substituting (11) into (10), we have

$$
\overline{\mathbf{S}}=2 \psi_{\mathrm{g}}^{\prime}\left(\bar{I}_{1}\right) \mathbf{I}+\alpha \mathbf{E}_{1} \otimes \mathbf{E}_{1}+\beta \mathbf{E}_{2} \otimes \mathbf{E}_{2}+\gamma\left(\mathbf{E}_{1} \otimes \mathbf{E}_{2}+\mathbf{E}_{2} \otimes \mathbf{E}_{1}\right)
$$

where

$$
\begin{aligned}
& \alpha=\frac{2}{\pi} \int_{\Sigma} \rho(\Theta) \psi_{\mathrm{n}}^{\prime}\left(\bar{I}_{4}(\Theta)\right) \cos ^{2} \Theta \mathrm{d} \Theta, \\
& \beta=\frac{2}{\pi} \int_{\Sigma} \rho(\Theta) \psi_{\mathrm{n}}^{\prime}\left(\bar{I}_{4}(\Theta)\right) \sin ^{2} \Theta \mathrm{d} \Theta, \\
& \gamma=\frac{2}{\pi} \int_{\Sigma} \rho(\Theta) \psi_{\mathrm{n}}^{\prime}\left(\bar{I}_{4}(\Theta)\right) \sin \Theta \cos \Theta \mathrm{d} \Theta .
\end{aligned}
$$

The Cauchy stress tensor $\boldsymbol{\sigma}=J^{-1} \mathbf{F S F}^{\mathrm{T}}$ is defined as the push-forward of the second PiolaKirchhoff stress tensor $\mathbf{S}$, and its fictitious isochoric part is given by

$$
\overline{\boldsymbol{\sigma}}=J^{-1} \overline{\mathbf{F}} \overline{\mathbf{S}} \overline{\mathbf{F}}^{\mathrm{T}}=J^{-1}\left[2 \psi_{\mathrm{g}}^{\prime} \overline{\mathbf{b}}+\alpha \overline{\mathbf{e}}_{1} \otimes \overline{\mathbf{e}}_{1}+\beta \overline{\mathbf{e}}_{2} \otimes \overline{\mathbf{e}}_{2}+\gamma\left(\overline{\mathbf{e}}_{1} \otimes \overline{\mathbf{e}}_{2}+\overline{\mathbf{e}}_{2} \otimes \overline{\mathbf{e}}_{1}\right)\right],
$$

where $\overline{\mathbf{e}}_{i}=\overline{\mathbf{F}} \mathbf{E}_{i}, i=1,2$, and $\overline{\mathbf{b}}=\overline{\mathbf{F}} \overline{\mathbf{F}}^{\mathrm{T}}$ is the modified left Cauchy-Green tensor. The isochoric Cauchy stress tensor $\boldsymbol{\sigma}_{\text {iso }}$ is given by

$$
\boldsymbol{\sigma}_{\text {iso }}=\mathbb{P}: \overline{\boldsymbol{\sigma}}
$$

where $\mathbb{P}=\mathbb{I}-\frac{1}{3} \mathbf{I} \otimes \mathbf{I}$ is the fourth-order projection tensor in the Eulerian description and $\mathbb{I}$ is the symmetric Eulerian fourth-order identity tensor.

\subsubsection{Elasticity Tensor}

In a similar way to the decoupling of the stress, the decoupled form of the elasticity tensor $\mathbb{C}$ in the Eulerian description is given by [23], p. 265,

$$
\mathbb{C}=\mathbb{C}_{\mathrm{vol}}+\mathbb{C}_{\text {iso }}
$$

Again, since the volumetric part $\mathbb{C}_{\mathrm{vol}}$ of the elasticity tensor $\mathbb{C}$ has been well documented, here we confine attention only to the isochoric part, i.e. [23]

$$
\mathbb{C}_{\text {iso }}=J^{-1} \mathbb{P}: \overline{\mathbb{C}}: \mathbb{P}+\frac{2}{3} \operatorname{tr}(\overline{\boldsymbol{\sigma}}) \mathbb{P}-\frac{2}{3}\left(\boldsymbol{\sigma}_{\text {iso }} \otimes \mathbf{I}+\mathbf{I} \otimes \boldsymbol{\sigma}_{\text {iso }}\right),
$$

where $\overline{\mathbb{C}}$ is the fourth-order fictitious elasticity tensor in the Eulerian description which is obtained by a push-forward operation of its Lagrangian form

$$
\overline{\mathbb{C}}=2 J^{-4 / 3} \frac{\partial \overline{\mathbf{S}}}{\partial \overline{\mathbf{C}}}=2 J^{-4 / 3} \frac{\partial \overline{\mathbf{S}}}{\partial \bar{I}_{1}} \otimes \mathbf{I}+2 J^{-4 / 3} \frac{\partial \overline{\mathbf{S}}}{\partial \bar{I}_{4}} \otimes(\mathbf{N} \otimes \mathbf{N}) .
$$


Substituting $\overline{\mathbf{S}}$ from (10) into (20), we obtain,

$$
\overline{\mathbb{C}}=4 J^{-4 / 3} \psi_{\mathrm{g}}^{\prime \prime}\left(\bar{I}_{1}\right) \mathbf{I} \otimes \mathbf{I}+J^{-4 / 3} \frac{4}{\pi} \int_{\Sigma} \rho(\Theta) \psi_{\mathrm{n}}^{\prime \prime}\left(\bar{I}_{4}(\Theta)\right) \mathbf{N} \otimes \mathbf{N} \otimes \mathbf{N} \otimes \mathbf{N} \mathrm{d} \Theta,
$$

where we have used the definitions

$$
\psi_{\mathrm{g}}^{\prime \prime}\left(\bar{I}_{1}\right)=\frac{\partial^{2} \Psi_{\mathrm{g}}\left(\bar{I}_{1}\right)}{\partial \bar{I}_{1} \partial \bar{I}_{1}}, \quad \psi_{\mathrm{n}}^{\prime \prime}\left(\bar{I}_{4}(\Theta)\right)=\frac{\partial^{2} \Psi_{\mathrm{n}}\left(\bar{I}_{4}(\Theta)\right)}{\partial \bar{I}_{4} \partial \bar{I}_{4}} .
$$

For the neo-Hookean strain-energy function and the standard fiber reinforcing model (5), we have

$$
\overline{\mathbb{C}}=J^{-4 / 3} \frac{4 \nu}{\pi} \int_{\Sigma} \rho(\Theta) \mathbf{N} \otimes \mathbf{N} \otimes \mathbf{N} \otimes \mathbf{N} \mathrm{d} \Theta .
$$

A push-forward operation yields the Eulerian fourth-order fictitious elasticity tensor as

$$
\overline{\mathbb{C}}=\frac{4 \nu}{\pi} \int_{\Sigma} \rho(\Theta) \overline{\mathbf{n}} \otimes \overline{\mathbf{n}} \otimes \overline{\mathbf{n}} \otimes \overline{\mathbf{n}} \mathrm{d} \Theta
$$

where $\overline{\mathbf{n}}=\overline{\mathbf{F}} \mathbf{N}$.

\subsubsection{Boundary of the Integration Domain}

In this section we outline the formulation for the integration domain $\Sigma$ appearing in the isochoric Cauchy stress tensor (17) via (10) and (16), and the elasticity tensor (24). Generally, for an arbitrary planar fiber orientation $\mathbf{N}, I_{4}$ is given by [15]

$$
I_{4}(\Theta)=C_{11} \cos ^{2} \Theta+C_{22} \sin ^{2} \Theta+C_{12} \sin 2 \Theta
$$

where $C_{i j}$ are the components of $\mathbf{C}$. The integration domain $\Sigma$ is the range of the angle $\Theta$ for which

$$
C_{11} \cos ^{2} \Theta+C_{22} \sin ^{2} \Theta+C_{12} \sin 2 \Theta>1 .
$$

This can be rearranged in various forms and will be used explicitly in the examples that follow in Section 4 and, in particular, it is resolved numerically with a modified Newton method, as is described on Section 2.2.

\subsection{D Fiber Dispersion Model}

In general the fiber dispersion is not planar so that a 3D fiber dispersion should be considered [4]. Collagen fibers may be dispersed around a mean direction, either rotationally symmetrically 


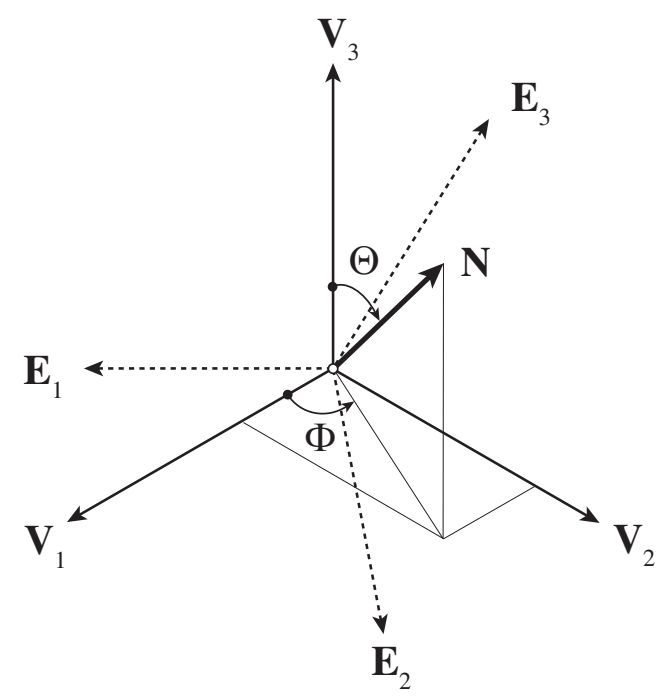

Figure 2: Arbitrary fiber orientation $\mathbf{N}$ (unit vector) within a 3D fiber dispersion described in the local coordinate system constructed by the eigenvectors $\mathbf{V}_{i}, i=1,2,3$, of the right CauchyGreen tensor $\mathbf{C}$. The components of $\mathbf{N}$ with respect to the global Cartesian basis $\mathbf{E}_{i}, i=1,2,3$, are determined by a rotation tensor $\mathbf{R}$ such that $\mathbf{V}_{i}=\mathbf{R} \mathbf{E}_{i}, i=1,2,3$, and $\mathbf{N}$ is given in terms of the eigenvectors by (27), where $\Theta$ and $\Phi$ are spherical polar angles.

or non-symmetrically, in the 3D space [14]. In this section, however, we assume for purposes of illustration that the fiber dispersion is rotationally symmetric. An arbitrary fiber direction $\mathbf{N}$ can be described by two angles $(\Theta, \Phi)$ as

$$
\mathbf{N}=\sin \Theta \cos \Phi \mathbf{V}_{1}+\sin \Theta \sin \Phi \mathbf{V}_{2}+\cos \Theta \mathbf{V}_{3}
$$

where $\mathbf{V}_{i}, i=1,2,3$, are the unit eigenvectors of $\mathbf{C}$, which for a given $\mathbf{C}$, define a unique local coordinate system, as depicted in Figure 2, where the two angles have the ranges $\Theta \in[0, \pi]$ and $\Phi \in[-\pi / 2, \pi / 2]$ which define a half sphere, denoted $\mathbb{S}$. The components of $\mathbf{N}$ in a global Cartesian coordinate system may be described by the basis vectors $\mathbf{E}_{i}, i=1,2,3$. Here we write $\mathbf{N}$ in terms of the eigenvectors of $\mathbf{C}$ instead of the global basis vectors because this makes it convenient for describing the integration boundary discussed in Section 2.3.3. Since the right Cauchy-Green tensor $\mathbf{C}$ is a Lagrangian deformation measure its eigenvectors are expressed as $\mathbf{V}_{i}=\mathbf{R E}_{i}, i=1,2,3$, where $\mathbf{R}$ is a rotation tensor (which depends on $\mathbf{C}$ ). Similar to the planar fiber dispersion model, we assume that the strain energy associated with the fiber in the direction $\mathbf{N}$ is a function of the fiber stretch in that direction, say $\Psi_{\mathrm{n}}\left(\bar{I}_{4}(\Theta, \Phi)\right)$. Now, in a slightly more general form than in [15], we write the total isochoric strain-energy function $\Psi_{\mathrm{f}}$ per unit volume in the reference configuration due to all the fibers using a PDF $\rho(\Theta, \Phi)$. We 
then obtain

$$
\Psi_{\mathrm{f}}=\frac{1}{2 \pi} \iint_{\Omega} \rho(\Theta, \Phi) \Psi_{\mathrm{n}}\left(\bar{I}_{4}(\Theta, \Phi)\right) \sin \Theta \mathrm{d} \Theta \mathrm{d} \Phi .
$$

In (28) we define $\bar{I}_{4}(\Theta, \Phi)=\overline{\mathbf{C}}: \mathbf{N} \otimes \mathbf{N}=J^{-2 / 3} I_{4}$, with $I_{4}=\mathbf{C}: \mathbf{N} \otimes \mathbf{N}$ and $\Omega=\{(\Theta, \Phi) \in$ $\left.\mathbb{S} \mid I_{4}(\Theta, \Phi)>1\right\}$ defines the region in which fibers are extended. In particular, for a rotationally symmetric fiber distribution $\rho$ is a function of the angle between $\mathbf{N}$ and the mean fiber direction which, as in Section 2.2, we denote by the unit vector M. For a 3D fiber dispersion, $\rho$ must satisfy the normalization condition

$$
\frac{1}{2 \pi} \iint_{\mathbb{S}} \rho(\Theta, \Phi) \sin \Theta \mathrm{d} \Theta \mathrm{d} \Phi=1
$$

Thus, the total isochoric strain-energy function of the material reads

$$
\Psi_{\text {iso }}=\Psi_{\mathrm{g}}\left(\bar{I}_{1}\right)+\frac{1}{2 \pi} \iint_{\Omega} \rho(\Theta, \Phi) \Psi_{\mathrm{n}}\left(\bar{I}_{4}(\Theta, \Phi)\right) \sin \Theta \mathrm{d} \Theta \mathrm{d} \Phi
$$

Note that it is straightforward to extend the current formulation to a non-symmetric dispersion model [14].

\subsubsection{Stress Tensors}

For a 3D fiber dispersion, the fictitious isochoric second Piola-Kirchhoff stress tensor, the counterpart of (10), becomes

$$
\overline{\mathbf{S}}=2 \psi_{\mathrm{g}}^{\prime} \mathbf{I}+\frac{1}{\pi} \iint_{\Omega} \rho(\Theta, \Phi) \psi_{\mathrm{n}}^{\prime}\left(\bar{I}_{4}(\Theta, \Phi)\right) \sin \Theta \mathbf{N} \otimes \mathbf{N} \mathrm{d} \Theta \mathrm{d} \Phi,
$$

where

$$
\begin{aligned}
\mathbf{N} \otimes \mathbf{N}= & \cos ^{2} \Theta \mathbf{V}_{3} \otimes \mathbf{V}_{3}+\sin ^{2} \Theta\left(\cos ^{2} \Phi \mathbf{V}_{1} \otimes \mathbf{V}_{1}+\sin ^{2} \Phi \mathbf{V}_{2} \otimes \mathbf{V}_{2}\right) \\
& +\sin \Theta \cos \Theta\left[\mathbf{V}_{3} \otimes\left(\mathbf{V}_{1} \cos \Phi+\mathbf{V}_{2} \sin \Phi\right)+\left(\mathbf{V}_{1} \cos \Phi+\mathbf{V}_{2} \sin \Phi\right) \otimes \mathbf{V}_{3}\right] \\
& +\sin ^{2} \Theta \sin \Phi \cos \Phi\left(\mathbf{V}_{1} \otimes \mathbf{V}_{2}+\mathbf{V}_{2} \otimes \mathbf{V}_{1}\right)
\end{aligned}
$$

Substituting (32) into (31), we obtain

$$
\begin{aligned}
\overline{\mathbf{S}}= & \psi_{\mathrm{g}}^{\prime}\left(\bar{I}_{1}\right) \mathbf{I}+\alpha \mathbf{V}_{3} \otimes \mathbf{V}_{3}+\beta \mathbf{V}_{1} \otimes \mathbf{V}_{1}+\beta^{\prime} \mathbf{V}_{2} \otimes \mathbf{V}_{2}+\gamma\left(\mathbf{V}_{3} \otimes \mathbf{V}_{1}+\mathbf{V}_{1} \otimes \mathbf{V}_{3}\right) \\
& +\gamma^{\prime}\left(\mathbf{V}_{2} \otimes \mathbf{V}_{3}+\mathbf{V}_{3} \otimes \mathbf{V}_{2}\right)+\delta\left(\mathbf{V}_{1} \otimes \mathbf{V}_{2}+\mathbf{V}_{2} \otimes \mathbf{V}_{1}\right),
\end{aligned}
$$


where

$$
\begin{aligned}
\alpha & =\frac{1}{\pi} \iint_{\Omega} \rho(\Theta, \Phi) \psi_{\mathrm{n}}^{\prime}\left(\bar{I}_{4}(\Theta, \Phi)\right) \cos ^{2} \Theta \sin \Theta \mathrm{d} \Theta \mathrm{d} \Phi \\
\beta & =\frac{1}{\pi} \iint_{\Omega} \rho(\Theta, \Phi) \psi_{\mathrm{n}}^{\prime}\left(\bar{I}_{4}(\Theta, \Phi)\right) \sin ^{3} \Theta \cos ^{2} \Phi \mathrm{d} \Theta \mathrm{d} \Phi \\
\beta^{\prime} & =\frac{1}{\pi} \iint_{\Omega} \rho(\Theta, \Phi) \psi_{\mathrm{n}}^{\prime}\left(\bar{I}_{4}(\Theta, \Phi)\right) \sin ^{3} \Theta \sin ^{2} \Phi \mathrm{d} \Theta \mathrm{d} \Phi \\
\gamma & =\frac{1}{\pi} \iint_{\Omega} \rho(\Theta, \Phi) \psi_{\mathrm{n}}^{\prime}\left(\bar{I}_{4}(\Theta, \Phi)\right) \cos \Theta \sin ^{2} \Theta \cos \Phi \mathrm{d} \Theta \mathrm{d} \Phi \\
\gamma^{\prime} & =\frac{1}{\pi} \iint_{\Omega} \rho(\Theta, \Phi) \psi_{\mathrm{n}}^{\prime}\left(\bar{I}_{4}(\Theta, \Phi)\right) \cos \Theta \sin ^{2} \Theta \sin \Phi \mathrm{d} \Theta \mathrm{d} \Phi \\
\delta & =\frac{1}{\pi} \iint_{\Omega} \rho(\Theta, \Phi) \psi_{\mathrm{n}}^{\prime}\left(\bar{I}_{4}(\Theta, \Phi)\right) \sin ^{3} \Theta \sin \Phi \cos \Phi \mathrm{d} \Theta \mathrm{d} \Phi
\end{aligned}
$$

Note that $\alpha, \beta$ and $\gamma$ are different here from those defined in (13) to (15). Note also that the formulas (37) to (39) correct the formulas (98) to (100) in [15] which contain typos in the exponent of $\sin \Theta$. A push-forward operation yields the fictitious isochoric Cauchy stress tensor $\overline{\boldsymbol{\sigma}}=J^{-1} \overline{\mathbf{F}} \overline{\mathbf{S}} \overline{\mathbf{F}}^{\mathrm{T}}$, which gives

$$
\begin{aligned}
\overline{\boldsymbol{\sigma}}= & J^{-1}\left[2 \psi_{\mathrm{g}}^{\prime} \overline{\mathbf{b}}+\alpha \overline{\mathbf{v}}_{3} \otimes \overline{\mathbf{v}}_{3}+\beta \overline{\mathbf{v}}_{1} \otimes \overline{\mathbf{v}}_{1}+\beta^{\prime} \overline{\mathbf{v}}_{2} \otimes \overline{\mathbf{v}}_{2}+\gamma\left(\overline{\mathbf{v}}_{3} \otimes \overline{\mathbf{v}}_{1}+\overline{\mathbf{v}}_{1} \otimes \overline{\mathbf{v}}_{3}\right)\right. \\
& \left.+\gamma^{\prime}\left(\overline{\mathbf{v}}_{2} \otimes \overline{\mathbf{v}}_{3}+\overline{\mathbf{v}}_{3} \otimes \overline{\mathbf{v}}_{2}\right)+\delta\left(\overline{\mathbf{v}}_{1} \otimes \overline{\mathbf{v}}_{2}+\overline{\mathbf{v}}_{2} \otimes \overline{\mathbf{v}}_{1}\right)\right]
\end{aligned}
$$

where $\overline{\mathbf{v}}_{i}=\overline{\mathbf{F}} \mathbf{V}_{i}, i=1,2,3$, are modified eigenvectors of the left Cauchy-Green tensor $\mathbf{b}$ (but not in general unit vectors). We then obtain the isochoric Cauchy stress tensor $\sigma_{\text {iso }}=$ $\mathbb{P}: \bar{\sigma}$, which has the same structure as in (17) but is now three dimensional rather than two dimensional.

\subsubsection{Elasticity Tensor}

A further differentiation of the fictitious second Piola-Kirchhoff stress tensor (31) with respect to $\overline{\mathbf{C}}$ yields the fourth-order fictitious elasticity tensor $\overline{\mathbb{C}}$ in the Lagrangian description as [23]

$$
\overline{\mathbb{C}}=4 J^{-4 / 3} \psi_{\mathrm{g}}^{\prime \prime}\left(\bar{I}_{1}\right) \mathbf{I} \otimes \mathbf{I}+J^{-4 / 3} \frac{2}{\pi} \int_{\Omega} \rho(\Theta, \Phi) \psi_{\mathrm{n}}^{\prime \prime}\left(\bar{I}_{4}(\Theta, \Phi)\right) \mathbf{N} \otimes \mathbf{N} \otimes \mathbf{N} \otimes \mathbf{N} \sin \Theta \mathrm{d} \Theta \mathrm{d} \Phi,
$$

where we have used the definition $(2.3)_{1}$ and

$$
\psi_{\mathrm{n}}^{\prime \prime}\left(\bar{I}_{4}(\Theta, \Phi)\right)=\frac{\partial^{2} \Psi_{\mathrm{n}}\left(\bar{I}_{4}(\Theta, \Phi)\right)}{\partial \bar{I}_{4} \partial \bar{I}_{4}} .
$$


For the neo-Hookean strain-energy function and the reinforcing model (5), we have

$$
\overline{\mathbb{C}}=J^{-4 / 3} \frac{2 \nu}{\pi} \iint_{\Omega} \rho(\Theta, \Phi) \mathbf{N} \otimes \mathbf{N} \otimes \mathbf{N} \otimes \mathbf{N} \sin \Theta \mathrm{d} \Theta \mathrm{d} \Phi .
$$

A push-forward operation gives the Eulerian fourth-order fictitious elasticity tensor

$$
\overline{\mathbb{C}}=\frac{2 \nu}{\pi} \iint_{\Omega} \rho(\Theta, \Phi) \overline{\mathbf{n}} \otimes \overline{\mathbf{n}} \otimes \overline{\mathbf{n}} \otimes \overline{\mathbf{n}} \sin \Theta \mathrm{d} \Theta \mathrm{d} \Phi .
$$

On substitution of (44) into (19) the complete form of the isochoric part of the elasticity tensor in the Eulerian description can be obtained but is not written explicitly here.

\subsubsection{Boundary of the Integration Domain}

With respect to its eigenvectors, $\mathbf{C}$ can be decomposed in the spectral form

$$
\mathbf{C}=\lambda_{1}^{2} \mathbf{V}_{1} \otimes \mathbf{V}_{1}+\lambda_{2}^{2} \mathbf{V}_{2} \otimes \mathbf{V}_{2}+\lambda_{3}^{2} \mathbf{V}_{3} \otimes \mathbf{V}_{3}
$$

where the squared principal stretches $\lambda_{i}^{2}, i=1,2,3$, are the eigenvalues of $\mathbf{C}$. Then, the invariant $I_{4}$ is given by [15]

$$
I_{4}(\Theta, \Phi)=\sin ^{2} \Theta\left(\lambda_{1}^{2} \cos ^{2} \Phi+\lambda_{2}^{2} \sin ^{2} \Phi\right)+\lambda_{3}^{2} \cos ^{2} \Theta .
$$

Generally, for any given deformation, we can label the principal stretches so that they are ordered as $\lambda_{1} \geq \lambda_{2} \geq \lambda_{3}$. For an incompressible material $\left(\lambda_{1} \lambda_{2} \lambda_{3}=1\right)$, the case $\lambda_{1}=\lambda_{2}=\lambda_{3}$ represents the reference configuration so that in general only two of the three principal stretches may be equal to each other. The integration domain $\Omega$ for the (3D) isochoric Cauchy stress tensor (17) with (34)-(39) and the Eulerian elasticity tensor (44) is the part of the half unit sphere $\mathbb{S}$ for which $I_{4}>1$, i.e.

$$
\sin ^{2} \Theta\left(\lambda_{1}^{2} \cos ^{2} \Phi+\lambda_{2}^{2} \sin ^{2} \Phi\right)+\lambda_{3}^{2} \cos ^{2} \Theta>1
$$

Because of the assumed ordering of the stretches, $\lambda_{3}$ is always smaller than 1 for incompressible materials, and $\lambda_{1}$ must be greater than 1 . By rearranging (47), we have the inequalities

$$
\left[\left(\lambda_{1}^{2}-\lambda_{3}^{2}\right)-\left(\lambda_{1}^{2}-\lambda_{2}^{2}\right) \sin ^{2} \Phi\right] \sin ^{2} \Theta>1-\lambda_{3}^{2}>0 .
$$

It follows that $\sin ^{2} \Theta \neq 0$ and $\Theta \neq n \pi, n=0,1$, and also

$$
\left(\lambda_{1}^{2}-\lambda_{3}^{2}\right)-\left(\lambda_{1}^{2}-\lambda_{2}^{2}\right) \sin ^{2} \Phi>0 \text {. }
$$

We now evaluate (49) for the cases $\lambda_{1}=\lambda_{2}$ and $\lambda_{1}>\lambda_{2}$. 


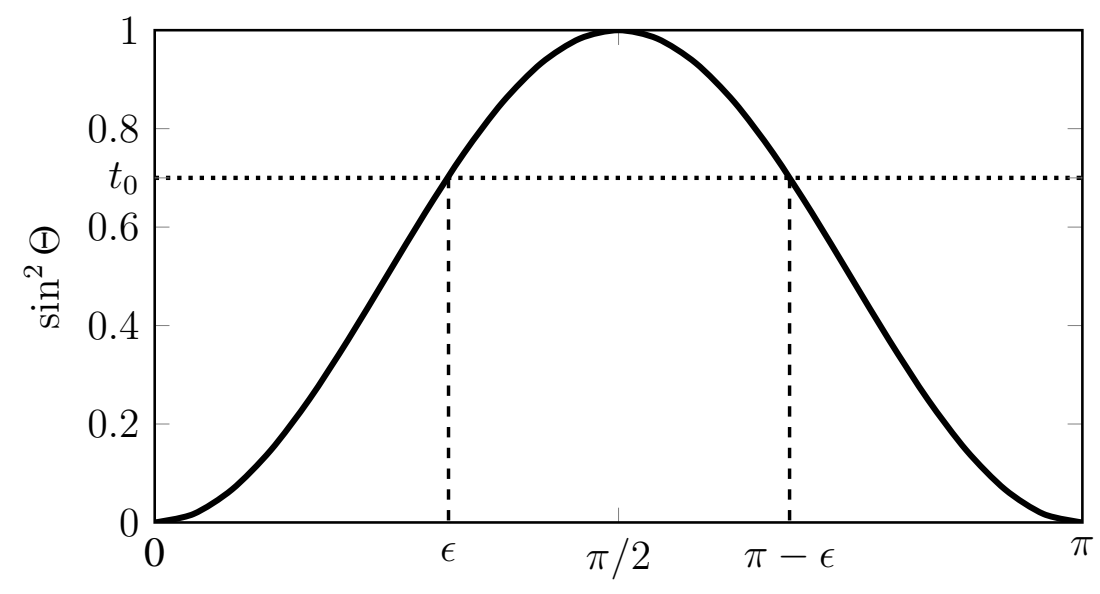

Angle $\Theta$

Figure 3: Plot of the function $\sin ^{2} \Theta$ between $\Theta=0$ and $\pi$. For a given value of $t=t_{0} \in(0,1)$, $\sin ^{2} \Theta>t_{0}$ above the dotted line, and $\Theta=\varepsilon$ or $\pi-\varepsilon$ where $\sin ^{2} \varepsilon=t_{0}$.

- If $\lambda_{1}=\lambda_{2}$, then (49) is satisfied for any $\Phi$. In addition, from (48) we have

$$
\sin ^{2} \Theta>\frac{1-\lambda_{3}^{2}}{\lambda_{1}^{2}-\lambda_{3}^{2}}=t, \quad 0<t<1,
$$

which indicates that $\Theta \in(\varepsilon, \pi-\varepsilon)$ for some $\varepsilon \in(0, \pi / 2)$; see Figure 3 .

- If $\lambda_{1}>\lambda_{2}$, we have

$$
\frac{\lambda_{1}^{2}-\lambda_{3}^{2}}{\lambda_{1}^{2}-\lambda_{2}^{2}}>\sin ^{2} \Phi
$$

If $\lambda_{2}>\lambda_{3}$, then (51) and (49) are satisfied for any $\Phi$. However, if $\lambda_{3}=\lambda_{2}$, then $\Phi \neq n \pi / 2, n=-1,1$.

From (48), we can also conclude that the integration domain $\Omega$ is symmetric about $\Theta=\pi / 2$ because $\sin ^{2} \Theta=\sin ^{2}(\pi-\Theta)$. Now if we let $\Theta=\pi / 2$, then $\sin ^{2} \Theta=1$ and (48) becomes

$$
\lambda_{1}^{2}-\left(\lambda_{1}^{2}-\lambda_{2}^{2}\right) \sin ^{2} \Phi>1
$$

If $\lambda_{1}=\lambda_{2}$, then (52) is satisfied for any $\Phi$. If $\lambda_{1}>\lambda_{2}$, then

$$
\sin ^{2} \Phi<\frac{\lambda_{1}^{2}-1}{\lambda_{1}^{2}-\lambda_{2}^{2}}=u, \quad u>0 .
$$

Because $u$ is positive, there always exists a range of values of $\Phi$ for which (53) is satisfied and $I_{4}>1$ when $\Theta=\pi / 2$. Based on this observation, we can always calculate the values of $\Phi$ at the boundary of the integration domain when $\Theta=\pi / 2$, as illustrated in Figure 4. Indeed, from 


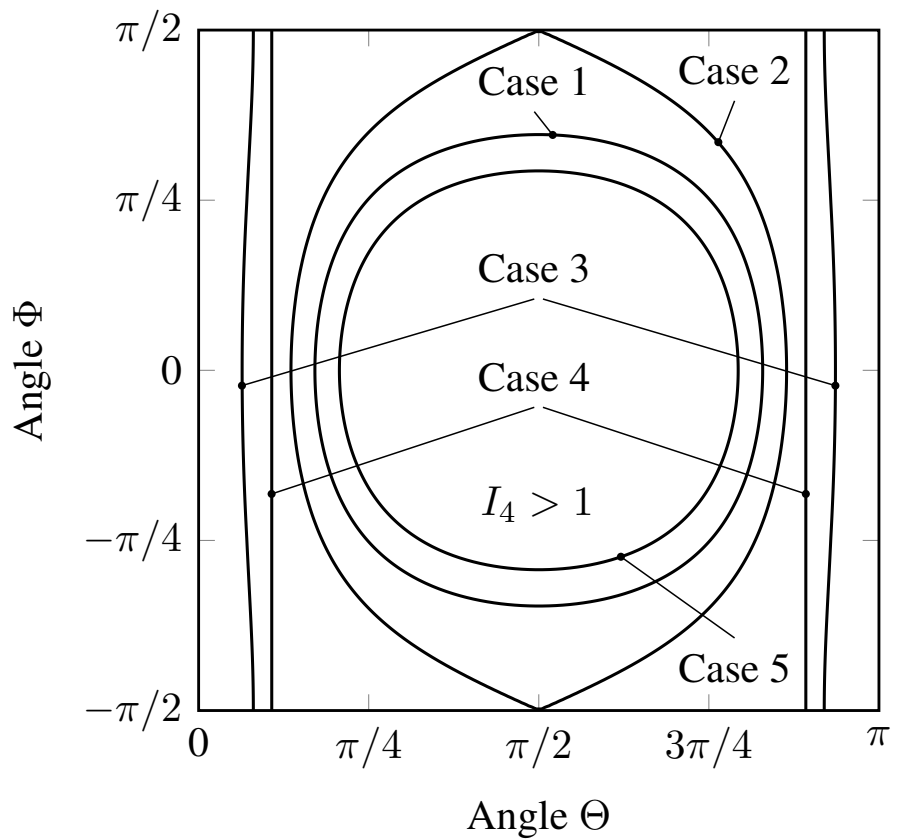

Figure 4: Illustration of the five cases of the integration domain within the $(\Theta, \Phi)$-space for the 3D fiber dispersion model with the following principal stretches. Case 1: $\lambda_{1}=1.35, \lambda_{2}=0.88$, $\lambda_{3}=0.8417$; Case 2: $\lambda_{1}=2.2, \lambda_{2}=1.0, \lambda_{3}=0.4545$; Case 3: $\lambda_{1}=5.0, \lambda_{2}=4.0$, $\lambda_{3}=0.005$; Case 4: $\lambda_{1}=3.0, \lambda_{2}=3.0, \lambda_{3}=0.1111$; Case 5: $\lambda_{1}=1.001, \lambda_{2}=0.9995$, $\lambda_{3}=0.9995$.

(48), it is easy to show that $\Phi$ has its peak values, say $\pm \Phi_{c}$, when $\Theta=\pi / 2$. Moreover, as well as the integration domain $\Omega$ being symmetric about $\Theta=\pi / 2$, it is symmetric about $\Phi=0$. There is always a range of $\Phi \in\left[-\Phi_{\mathrm{c}}, \Phi_{\mathrm{c}}\right]$ when $\Theta=\pi / 2$ for which $I_{4}>1$. For any given value of $\Phi$ within the integration domain there exist at most two angles of $\Theta$ on the boundary of $\Omega$.

Here it is only necessary to search the integration domain $\Omega$ within one half of the sphere, because the directions $\mathbf{N}$ and $-\mathbf{N}$ represent the same fiber. Note that the fiber directions for which $\Theta=0$ and $\Theta=\pi$ are always outside the integration domain. In summary, there are five cases to consider depending on the relative distributions of the stretches $\lambda_{i}, i=1,2,3$ :

- Case 1: $\lambda_{1}>1>\lambda_{2}>\lambda_{3}$

When $\Theta=\pi / 2$, the two values of $\Phi$ on the boundary of the integration domain can be determined from

$$
\sin ^{2} \Phi=\frac{\lambda_{1}^{2}-1}{\lambda_{1}^{2}-\lambda_{2}^{2}}=u, \quad 0<u<1,
$$

so that $\Phi \in\left[-\Phi_{\mathrm{c}}, \Phi_{\mathrm{c}}\right]$, where

$$
\Phi_{\mathrm{c}}=\arcsin \left(u^{1 / 2}\right)<\frac{\pi}{2}
$$


When $\Theta \neq \pi / 2$, then for each $\Phi \in\left[-\Phi_{c}, \Phi_{c}\right]$, we can determine the values of $\Theta$ on the integration boundary by using $(48)_{1}$ with the inequality replaced by equailty.

- Case 2: $\lambda_{1}>1=\lambda_{2}>\lambda_{3}$

Similar to Case 1 except that $\Phi_{\mathrm{c}}=\pi / 2$.

- Case 3: $\lambda_{1}>\lambda_{2}>1>\lambda_{3}$

This is similar to Case 1, except that $\Phi_{\mathrm{c}}=\pi / 2$, because attention is restricted to the range $\Phi \in[-\pi / 2, \pi / 2]$.

- Case 4: $\lambda_{1}=\lambda_{2}>1>\lambda_{3}$

Now (48) is satisfied for any $\Phi \in[-\pi / 2, \pi / 2]$ and reduces to

$$
\sin ^{2} \Theta>\frac{1-\lambda_{3}^{2}}{\lambda_{1}^{2}-\lambda_{3}^{2}}=t, \quad 0<t<1,
$$

so that $\Theta \in\left[\Theta_{c}, \pi-\Theta_{c}\right]$, where

$$
\Theta_{\mathrm{c}}=\arcsin \left(t^{1 / 2}\right)<\frac{\pi}{2} .
$$

For this special case the integration domain becomes a rectangle, as shown in Figure 4.

- Case 5: $\lambda_{1}>1>\lambda_{2}=\lambda_{3}$

Now (48) becomes

$$
\left(\lambda_{1}^{2}-\lambda_{3}^{2}\right) \cos ^{2} \Phi \sin ^{2} \Theta>1-\lambda_{3}^{2}>0 .
$$

When $\Theta=\pi / 2$, the boundary of the integration domain is given by

$$
\cos ^{2} \Phi=\frac{1-\lambda_{3}^{2}}{\lambda_{1}^{2}-\lambda_{3}^{2}}=t, \quad 0<t<1,
$$

so that $\Phi \in\left[-\Phi_{\mathrm{c}}, \Phi_{\mathrm{c}}\right]$, as exemplified in Figure 5, where

$$
\Phi_{\mathrm{c}}=\arccos \left(t^{1 / 2}\right)<\frac{\pi}{2} .
$$

For each $\Phi \in\left[-\Phi_{\mathrm{c}}, \Phi_{\mathrm{c}}\right]$ the values of $\Theta$ on the integration boundary are determined from $(58)_{1}$ with the inequality replaced by equality.

\section{Finite Element Implementation}

The proposed 2D and 3D fiber dispersion models have been implemented in the general purpose finite element analysis program FEAP [27] at the integration point level. In this section, we present the details of the computational implementation for both 2D and 3D models. 


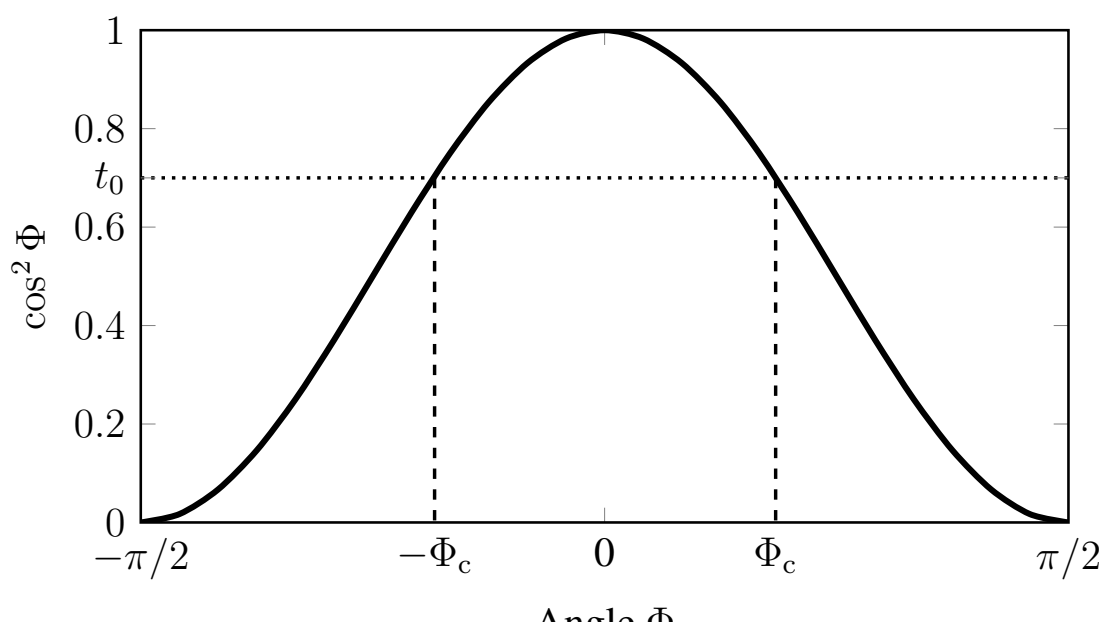

Angle $\Phi$

Figure 5: Plot of the function $\cos ^{2} \Phi$ for $\Phi$ between $-\pi / 2$ and $\pi / 2$. For a given value $t=t_{0} \in$ $(0,1), \cos ^{2} \Phi>t_{0}$ above the dotted line and $\Phi= \pm \Phi_{\mathrm{c}}$, where $\cos \Phi_{\mathrm{c}}=\sqrt{t_{0}}$.

\subsection{Planar Fiber Dispersion Model}

To implement the planar fiber dispersion model, for example, we can choose a von Mises distribution [15] as the PDF, i.e.

$$
\rho(\Theta)=4 \sqrt{\frac{b}{2 \pi}} \frac{\exp \left[2 b \cos ^{2}\left(\Theta-\Theta_{\mathrm{M}}\right)\right]}{\operatorname{erfi}(\sqrt{2 b})},
$$

where $b$ is a constant concentration parameter and erfi $(x)=-\mathrm{i} \operatorname{erf}(\mathrm{i} x)$ denotes the imaginary error function in which the error function $\operatorname{erf}(x)$ is defined by

$$
\operatorname{erf}(x)=\frac{2}{\sqrt{\pi}} \int_{0}^{x} \exp \left(-\xi^{2}\right) \mathrm{d} \xi
$$

Substituting (61) into the scalar coefficients of the isochoric Cauchy stress tensor (17), with (13)-(16) for the standard reinforcing model, and the Eulerian fictitious elasticity tensor (24) and, by further expanding the term $\overline{\mathbf{n}} \otimes \overline{\mathbf{n}} \otimes \overline{\mathbf{n}} \otimes \overline{\mathbf{n}}$ in (24), we find that the scalar coefficients therein involve integrals of the form

$$
4 \sqrt{\frac{b}{2 \pi}} \frac{1}{\operatorname{erfi}(\sqrt{2 b})} \int_{\Sigma} \exp \left[2 b \cos ^{2}\left(\Theta-\Theta_{\mathrm{M}}\right)\right] \sin ^{i} \Theta \cos ^{j} \Theta \mathrm{d} \Theta,
$$

where $i, j \in\{0,1,2,3,4\}$. This integration will be evaluated numerically using a regular Gauss-Kronrod quadrature rule. A summary of the algorithm used to determine the integration domain for the planar fiber dispersion is shown in the accompanying box (Algorithm 1). It 


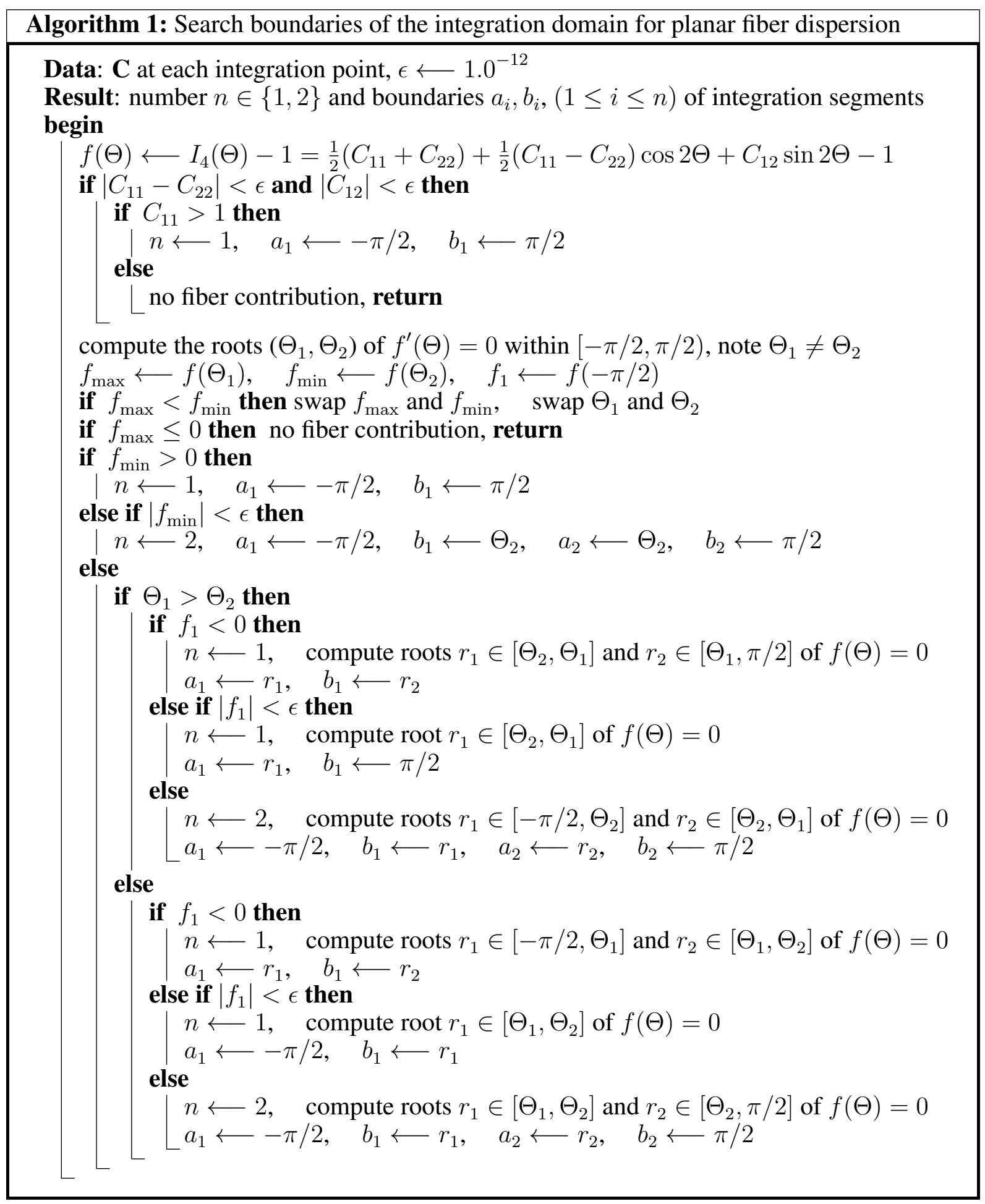

is necessary to use this procedure before applying Newton's method to find the roots of $I_{4}=1$. 


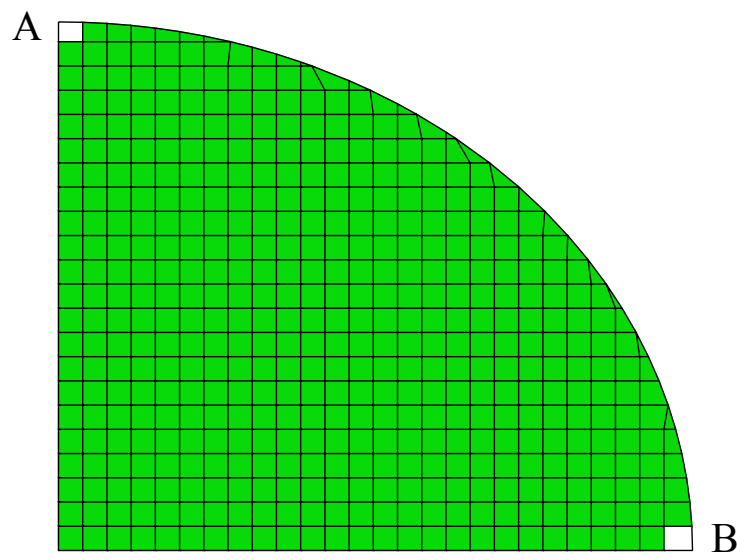

Figure 6: Representative example of an integration domain (one quarter shown) meshed with square, triangular and general quadrilateral elements. The major and minor axes are divided into equal segments except for the two adjacent to the boundary labeled A and B.

\subsection{D Fiber Dispersion Model}

For the 3D fiber dispersion model, we can use the same PDF as in (61) but in a slightly different form, namely

$$
\rho(\Theta, \Phi)=4 \sqrt{\frac{b}{2 \pi}} \frac{\exp \left[2 b(\mathbf{N} \cdot \mathbf{M})^{2}\right]}{\operatorname{erfi}(\sqrt{2 b})} .
$$

Similarly to Section 3.1, we substitute (64) into the scalar coefficients of the (3D) isochoric Cauchy stress tensor (17) and the Eulerian fictitious elasticity tensor (44) with (34) to (40). The coefficients obtained by expanding the term $\overline{\mathbf{n}} \otimes \overline{\mathbf{n}} \otimes \overline{\mathbf{n}} \otimes \overline{\mathbf{n}}$ in (44) involve the integrals

$$
4 \sqrt{\frac{b}{2 \pi}} \frac{1}{\operatorname{erfi}(\sqrt{2 b})} \iint_{\Omega} \exp \left[2 b(\mathbf{N} \cdot \mathbf{M})^{2}\right] \sin ^{i} \Theta \cos ^{j} \Theta \sin ^{k} \Phi \cos ^{l} \Phi \mathrm{d} \Theta \mathrm{d} \Phi,
$$

where each of indices $i, j, k, l$ can be any one of the numbers in $\{0,1,2,3,4,5\}$. These integrals are evaluated numerically using an adaptive finite element integration scheme. Firstly, the integration domain determined in Section 2.3.3 for each integration point is discretized with square, triangular and general quadrilateral elements. Briefly, because of the symmetry, the integration domain is divided into four equal quarters. One quarter of the domain is discretized as follows: half of the major and minor axes of the domain are divided into equal segments except the two segments adjacent to the boundary (labeled A and B in Figure 6). The number of the segments is controlled by an internal parameter. Next all the nodes of the internal elements are determined and assembled into square elements. Then the rest of the nodes for the triangular and quadrilateral elements near the boundary are calculated. The resultant mesh, as shown in Figure 6 as a representative example, is mirrored in the other three quarters. 


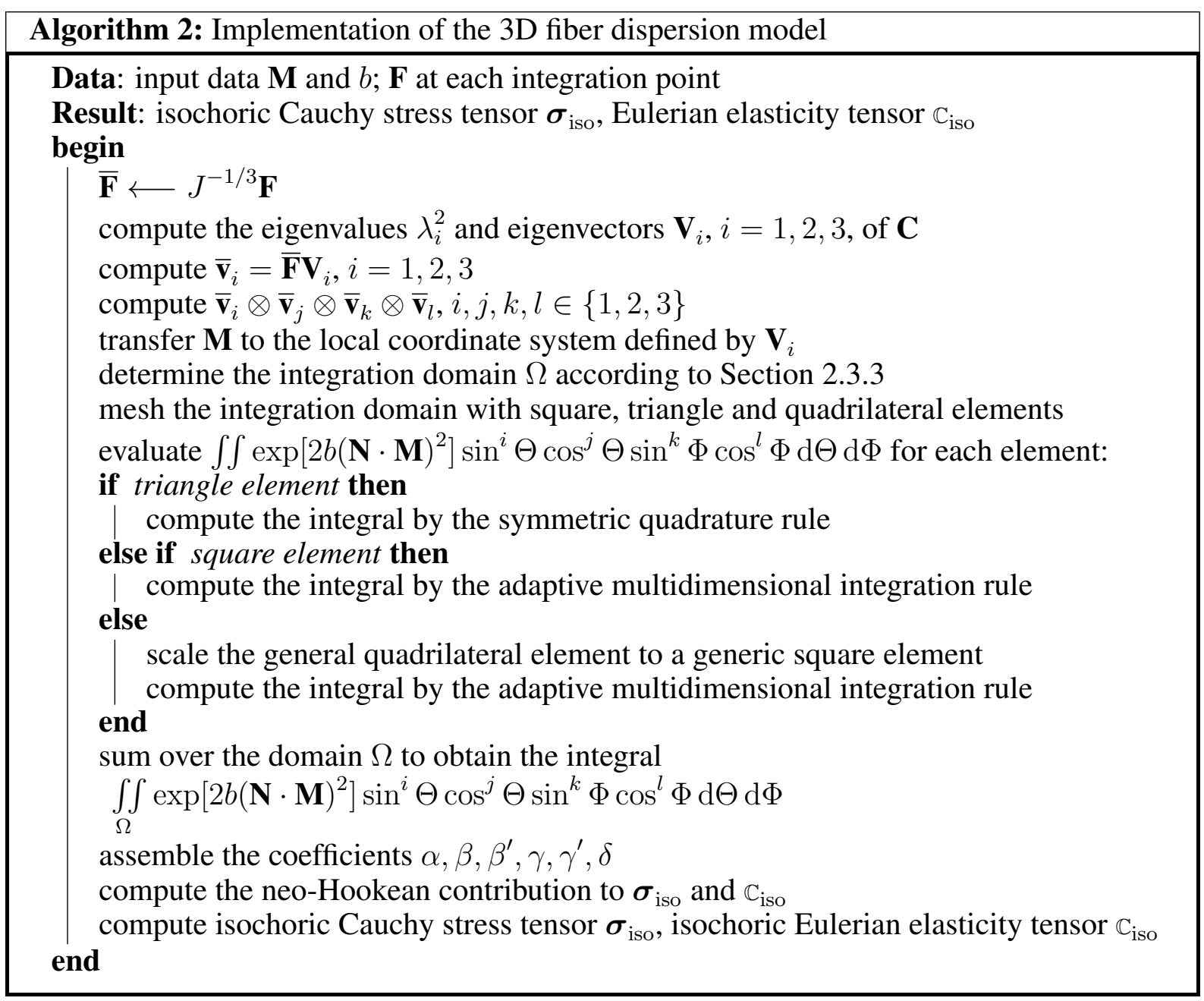

Secondly, we apply a global adaptive multidimensional integration rule [28, 29] on the square and quadrilateral elements, and a symmetric quadrature rule [30] on the triangular elements. Finally, a summation over all the elements yields the total integration (65) in the target domain. A general guideline to implement the proposed 3D fiber dispersion model is shown in the accompanying box (Algorithm 2).

\section{Representative Examples}

In this section we demonstrate the performance and the finite element implementation of the proposed continuum mechanical framework by means of representative numerical examples, specifically uniaxial extension and simple shear. Incompressible hyperelastic materials are assumed for all these examples. To enforce incompressibility, we have adopted the augmented Lagrangian method in FEAP [31]. In each example, the model geometry was discretized with 8- 


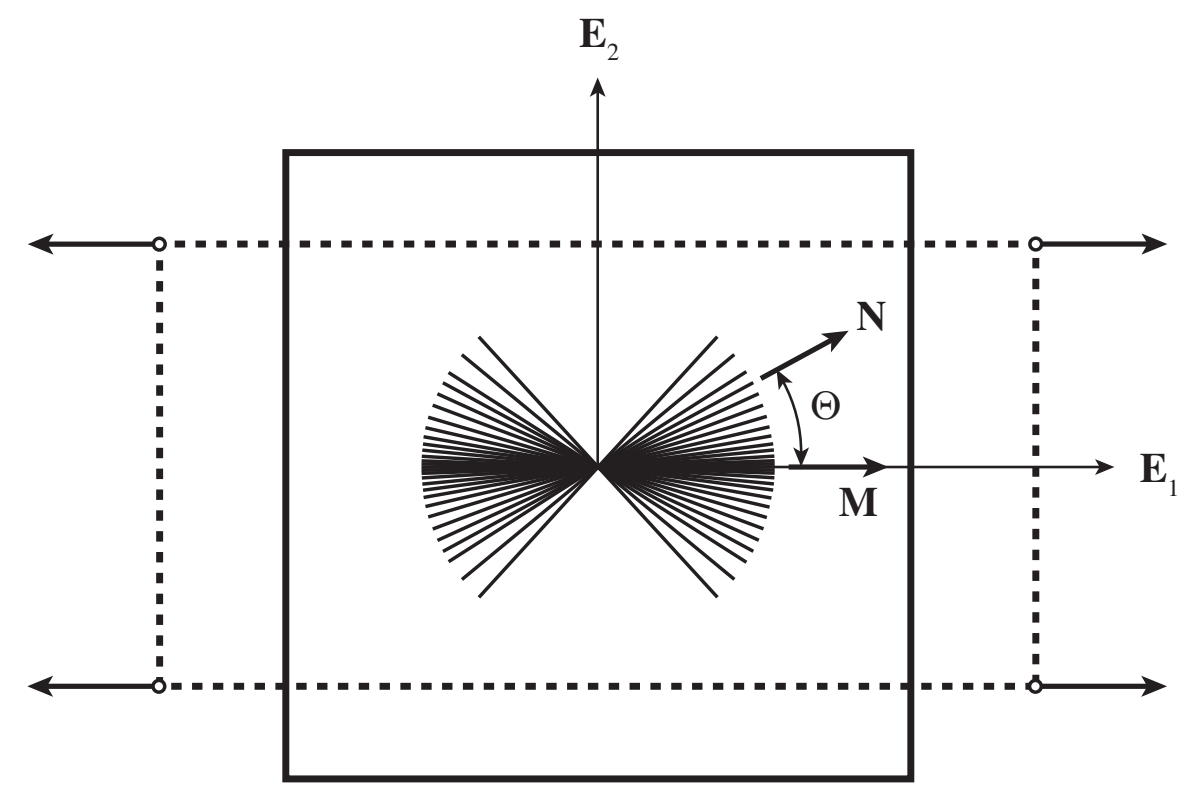

Figure 7: Cross-section of a unit cube element normal to $\mathbf{E}_{3}$ containing a planar fiber dispersion with mean fiber direction $\mathbf{M}$ where the loading direction is aligned along $\mathbf{E}_{1}$. An arbitrary fiber direction within the dispersion is given by $\mathbf{N}=\cos \Theta \mathbf{E}_{1}+\sin \Theta \mathbf{E}_{2}$. The dashed outline shows the deformed configuration.

node hexahedral Q1/P0 elements, and the problems were solved by using the Newton-Raphson method. Related analytical solutions obtained by using MATLAB or MATHEMATICA are presented to verify the simulation results.

\subsection{Planar Fiber Dispersion Model}

We start with a uniaxial extension example with loading in the mean fiber direction such that the deformation is homogeneous and the deformation gradient is diagonal. Then, we investigate a more general example in which the mean fiber direction is aligned in an arbitrary direction relative to the loading direction, which leads to a non-homogeneous deformation. Finally, simple shear examples with three different mean fiber orientations are presented.

\subsubsection{Uniaxial Extension in the Mean Fiber Direction}

In this first example we illustrate the efficacy of the planar fiber dispersion model by using a uniaxial extension test in the mean fiber direction. The model geometry (discretized by one hexahedral element) is $1 \times 1 \times 1 \mathrm{~mm}$ aligned with the axes $\mathbf{E}_{1}, \mathbf{E}_{2}$ and $\mathbf{E}_{3}$. One family of 
fibers is embedded in each cross-section of the cube normal to the $\mathbf{E}_{3}$ direction and assumed to be dispersed symmetrically about the mean fiber direction $\mathbf{E}_{1}$. The sample is subjected to unconfined uniaxial extension in the $\mathbf{E}_{1}$ direction such that the deformation is homogeneous. Thus, we have assumed that the mean fiber direction $\mathbf{M}$ and the loading direction are aligned with $\mathbf{E}_{1}$, as depicted in Figure 7.

The deformation gradient and the right Cauchy-Green tensor in matrix form are given by

$$
[\mathbf{F}]=\operatorname{diag}\left[\lambda_{1}, \lambda_{2}, \lambda_{3}\right], \quad[\mathbf{C}]=\operatorname{diag}\left[\lambda_{1}^{2}, \lambda_{2}^{2}, \lambda_{3}^{2}\right],
$$

where $\lambda_{i}, i=1,2,3$, is the stretch of the material in the direction $\mathbf{E}_{i}$. Hence, in the Eulerian description, the push-forwards of $\mathbf{E}_{1}, \mathbf{E}_{2}$ and $\mathbf{E}_{3}$, denoted $\mathbf{e}_{1}, \mathbf{e}_{2}$ and $\mathbf{e}_{3}$, have components

$$
\left[\mathbf{e}_{1}\right]=[\mathbf{F}]\left[\mathbf{E}_{1}\right]=\left[\lambda_{1}, 0,0\right], \quad\left[\mathbf{e}_{2}\right]=[\mathbf{F}]\left[\mathbf{E}_{2}\right]=\left[0, \lambda_{2}, 0\right], \quad\left[\mathbf{e}_{3}\right]=[\mathbf{F}]\left[\mathbf{E}_{3}\right]=\left[0,0, \lambda_{3}\right] .
$$

For an arbitrary (in-plane) fiber direction, $I_{4}(\Theta)$ is given by

$$
I_{4}(\Theta)=\lambda_{1}^{2} \cos ^{2} \Theta+\lambda_{2}^{2} \sin ^{2} \Theta .
$$

For an incompressible material the Cauchy stress tensor $\sigma$, the counterpart of the fictitious isochoric Cauchy stress (16), is

$$
\boldsymbol{\sigma}=-p \mathbf{I}+\mu \mathbf{b}+\alpha \mathbf{e}_{1} \otimes \mathbf{e}_{1}+\beta \mathbf{e}_{2} \otimes \mathbf{e}_{2}+\gamma\left(\mathbf{e}_{1} \otimes \mathbf{e}_{2}+\mathbf{e}_{2} \otimes \mathbf{e}_{1}\right)
$$

where $p$ is the Lagrange multiplier and

$$
\begin{aligned}
& \alpha=\frac{2 \nu}{\pi} \int_{\Sigma} \rho(\Theta)\left(I_{4}(\Theta)-1\right) \cos ^{2} \Theta \mathrm{d} \Theta, \\
& \beta=\frac{2 \nu}{\pi} \int_{\Sigma} \rho(\Theta)\left(I_{4}(\Theta)-1\right) \sin ^{2} \Theta \mathrm{d} \Theta, \\
& \gamma=\frac{2 \nu}{\pi} \int_{\Sigma} \rho(\Theta)\left(I_{4}(\Theta)-1\right) \cos \Theta \sin \Theta \mathrm{d} \Theta,
\end{aligned}
$$

in which the standard reinforcing model (5) has been adopted with $\bar{I}_{4}=I_{4}$. From (69) the component of the Cauchy stress tensor in the mean fiber direction, denoted $\sigma$, is

$$
\sigma=-p+(\mu+\alpha) \lambda^{2}
$$

where $\lambda=\lambda_{1}$ is the corresponding stretch. For this uniaxial extension test we take $\sigma_{22}=\sigma_{33}=$ 0 , and hence $p=(\mu+\beta) \lambda_{2}^{2}=\mu \lambda_{3}^{2}$. From the incompressibility condition $\lambda_{1} \lambda_{2} \lambda_{3}=1$ we then have

$$
\lambda_{2}^{2}=\sqrt{\frac{\mu}{\mu+\beta}} \lambda^{-1}
$$




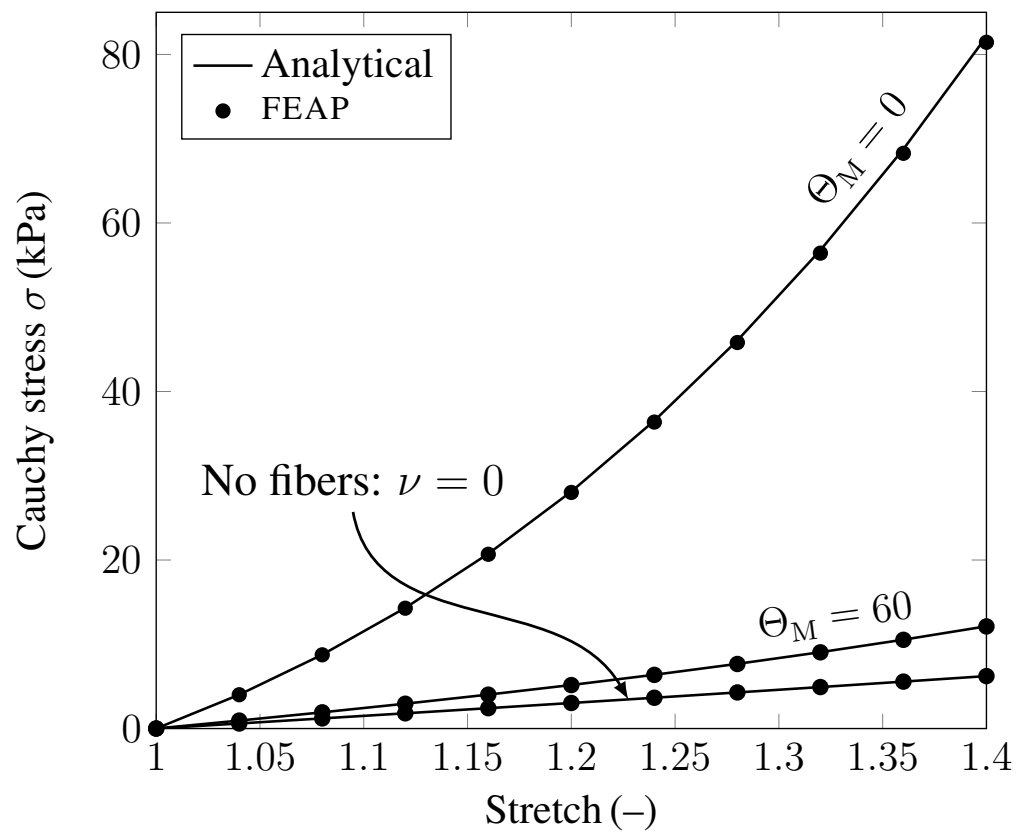

Figure 8: Comparison of FEAP results and the analytical solutions obtained using MATLAB for the uniaxial extension of a cube in the mean fiber direction $\left(\Theta_{M}=0\right)$, together with the analytical result in the absence of fibers $(\nu=0)$. The results for the angle $\Theta_{M}=60^{\circ}$ refer to the example in Section 4.1.2.

so that from (73) we obtain

$$
\sigma=(\mu+\alpha) \lambda^{2}-\sqrt{\mu(\mu+\beta)} \lambda^{-1}
$$

The numerical integrations of the coefficients $\alpha, \beta$ and $\gamma$ given by (70)-(72) were simplified using the expression (68) and then evaluated in MATLAB R2010b (The MathWorks Inc., Natick, MA, USA) with the adaptive Gauss-Kronrod quadrature method (quadgk). Note that $\alpha, \beta$ and $\gamma$ depend on $\lambda$. For comparison between the numerical computation obtained using FEAP and the analytical solutions obtained using MATLAB, we have used the material parameters $\mu=5 \mathrm{kPa}$, $\nu=10 \mathrm{kPa}$ and $b=2.9$. Figure 8 shows the results from FEAP together with the corresponding analytical results for which the stretches $\lambda$ and $\lambda_{2}$ are determined using FEAP at each load step. Also shown are the analytical and numerical results for $\nu=0$ (no fiber contribution). Here $\lambda_{2}$, given by (74), is also needed to compute the coefficients $\alpha$ and $\beta$ in (75). In Section 4.1.2 we consider a more general uniaxial extension case in which $\Theta_{M}$ is not equal to zero, and the deformation is inhomogeneous. In particular, the result for $\Theta_{M}=60^{\circ}$ is also shown in Figure 8, where in this case $\sigma=\sigma_{11}$, which is calculated as described in the following section. 


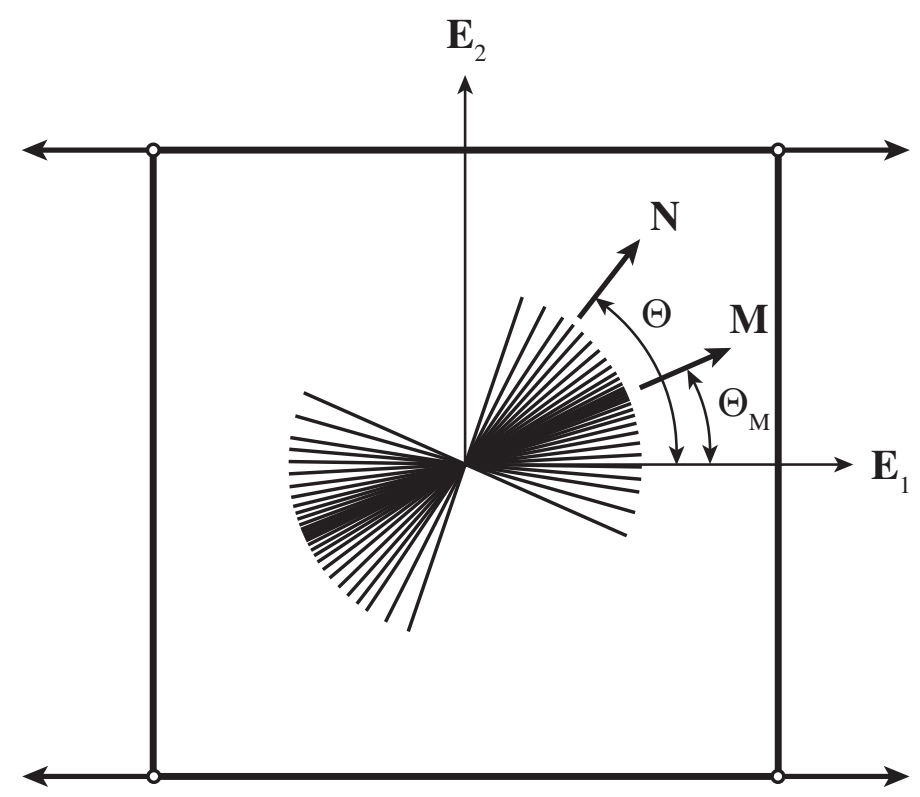

Figure 9: Cross-section of a unit cube element normal to $\mathbf{E}_{3}$ containing a planar fiber dispersion which is symmetric about the mean fiber direction $\mathbf{M}=\cos \Theta_{\mathrm{M}} \mathbf{E}_{1}+\sin \Theta_{\mathrm{M}} \mathbf{E}_{2}$. The loading direction is aligned along $\mathbf{E}_{1}$. An arbitrary fiber direction within the dispersion is given by $\mathbf{N}=\cos \Theta \mathbf{E}_{1}+\sin \Theta \mathbf{E}_{2}$.

\subsubsection{Planar Uniaxial Extension in a General Direction}

This is a similar example to that in the previous section except that we now consider a plane strain deformation in the $\left(\mathbf{E}_{1}, \mathbf{E}_{2}\right)$ plane with the mean fiber direction $\mathbf{M}$ aligned at an angle $\Theta_{\mathrm{M}}=60^{\circ}$ to the $\mathbf{E}_{1}$ direction, as illustrated by a representative unit cube element in Figure 9. The dispersion is assumed to be symmetric about $\mathbf{M}$. As a result the deformation is non-homogeneous and a $10 \times 4 \times 1 \mathrm{~mm}$ rectangular strip is considered for this example. The model geometry, as shown by the solid frame in Figure 10 is discretized by $20 \times 8 \times 2=320$ hexahedral elements (each element has dimensions of $0.5 \times 0.5 \times 0.5 \mathrm{~mm}$ ). Because the deformation is plane strain the front and back faces of the strip are fixed in the $\mathbf{E}_{3}$ direction. We also constrained all nodes of the bottom face of the model in the $\mathbf{E}_{1}$ direction and additionally the center node of the bottom face in the $\mathbf{E}_{2}$ and $\mathbf{E}_{3}$ directions. The node A of the left edge on the bottom face shown in Figure 10(a) is also constrained in the $\mathbf{E}_{3}$ direction to prevent rigid body rotation about $\mathbf{E}_{1}$.

The strip is subjected to a uniaxial stretch of $\lambda=1.4$ in the $\mathbf{E}_{1}$ direction applied on the top face. Because the fiber dispersion is non-symmetric about the loading direction and the deformation is plane strain the deformation gradient and the right Cauchy-Green tensor have 


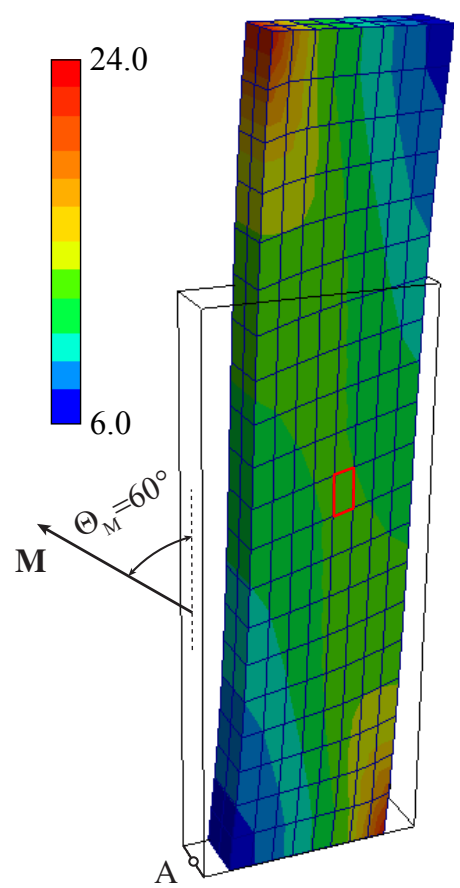

(a) $\sigma_{11}$

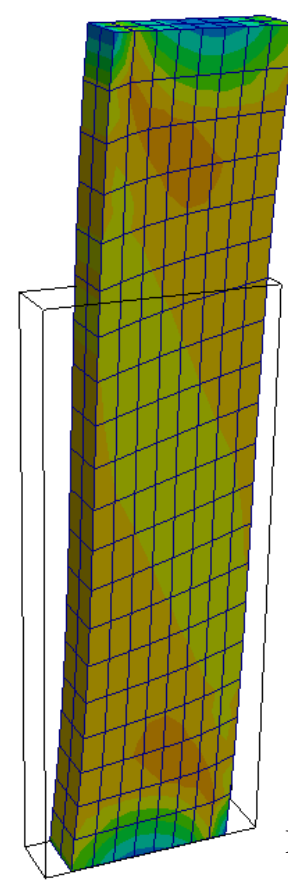

(b) $\sigma_{22}$

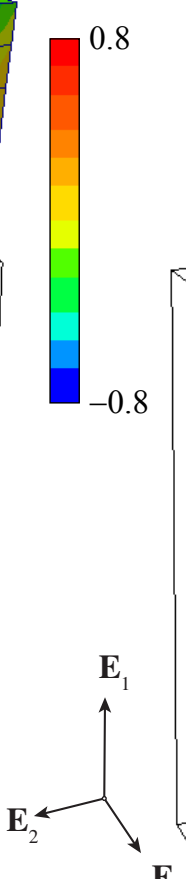

$\mathbf{E}_{3}$

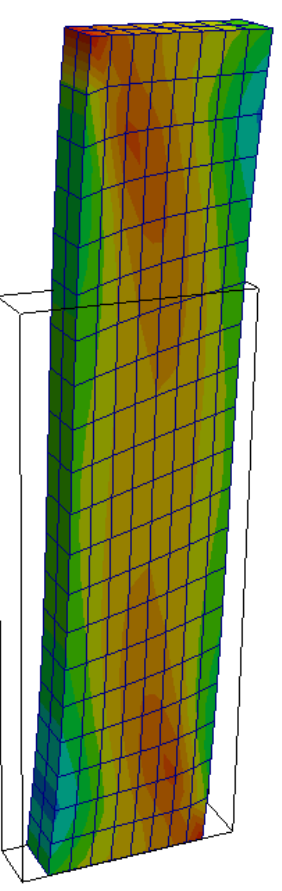

(c) $\sigma_{12}$

Figure 10: FEAP computation results for the uniaxial extension of a rectangular strip with the planar fiber dispersion model and a mean fiber angle $\Theta_{\mathrm{M}}=60^{\circ}$. The Cauchy stress distributions $\sigma_{11}, \sigma_{22}, \sigma_{12}$ in (a), (b), (c), respectively, are plotted for an axial stretch of $\lambda=1.4$ with parameters $\mu=5 \mathrm{kPa}, \nu=10 \mathrm{kPa}$ and $b=2.9$.

the matrix forms

$$
[\mathbf{F}]=\left[\begin{array}{ccc}
F_{11} & F_{12} & 0 \\
F_{21} & F_{22} & 0 \\
0 & 0 & 1
\end{array}\right], \quad[\mathbf{C}]=\left[\begin{array}{ccc}
C_{11} & C_{12} & 0 \\
C_{12} & C_{22} & 0 \\
0 & 0 & 1
\end{array}\right] .
$$

The incompressibility condition yields $F_{11} F_{22}-F_{12} F_{21}=1$. Then, $\mathbf{e}_{1}, \mathbf{e}_{2}$ and $\mathbf{e}_{3}$ have components

$$
\left[\mathbf{e}_{1}\right]=[\mathbf{F}]\left[\mathbf{E}_{1}\right]=\left[F_{11}, F_{21}, 0\right], \quad\left[\mathbf{e}_{2}\right]=[\mathbf{F}]\left[\mathbf{E}_{2}\right]=\left[F_{12}, F_{22}, 0\right], \quad\left[\mathbf{e}_{3}\right]=[\mathbf{F}]\left[\mathbf{E}_{3}\right]=[0,0,1],
$$

while $I_{4}$ is given by (68). From (69) the non-zero components of the Cauchy stress tensor are

$$
\begin{aligned}
& \sigma_{11}=-p+(\mu+\alpha) F_{11}^{2}+(\mu+\beta) F_{12}^{2}+2 \gamma F_{11} F_{12}, \\
& \sigma_{22}=-p+(\mu+\alpha) F_{21}^{2}+(\mu+\beta) F_{22}^{2}+2 \gamma F_{21} F_{22}, \\
& \sigma_{33}=-p+\mu \\
& \sigma_{12}=(\mu+\alpha) F_{11} F_{21}+(\mu+\beta) F_{12} F_{22}+\gamma\left(F_{11} F_{22}+F_{12} F_{21}\right) .
\end{aligned}
$$


Because the center node of the bottom face is fixed, the lateral stress component $\sigma_{22}$ is not zero near the top and bottom faces, but is approximately zero in the central region. Hence, we take $\sigma_{22}=0$ there, and then $p=(\mu+\alpha) F_{21}^{2}+(\mu+\beta) F_{22}^{2}+2 \gamma F_{21} F_{22}$, so that $\sigma_{11}$ and $\sigma_{33}$ become

$$
\begin{aligned}
& \sigma_{11}=(\mu+\alpha)\left(F_{11}^{2}-F_{21}^{2}\right)+(\mu+\beta)\left(F_{12}^{2}-F_{22}^{2}\right)+2 \gamma\left(F_{11} F_{12}-F_{21} F_{22}\right), \\
& \sigma_{33}=\mu-(\mu+\alpha) F_{21}^{2}-(\mu+\beta) F_{22}^{2}-2 \gamma F_{21} F_{22} .
\end{aligned}
$$

With the same material parameters as in Section 4.1.1, we now take $\Theta_{\mathrm{M}}=60^{\circ}$ (see also Figure 10(a).) Instead of the stretch $\lambda$ we output the components $F_{11}, F_{12}, F_{21}$ and $F_{22}$ of the deformation gradient from FEAP at one integration point of the central element identified by the red frame in Figure 10(a) for each increment, and then we performed an analytical calculation in MATLAB with the same numerical integration scheme used to evaluate $\alpha, \beta$ and $\gamma$. Because the deformation is non-homogeneous, we only verified the numerical results in the element indicated in the red frame. The boundary of the integration domain (26) was determined by using the fzero function in MATLAB. For the applied stretch at each increment the element stress in the loading direction output from FEAP is then compared with the corresponding MATLAB computation (82) and shown in Figure 8. As can be seen, and as expected, when the fibers are aligned away from the loading direction, the stress is reduced significantly.

For this non-homogeneous problem the spatial distributions of the stress components $\sigma_{11}$, $\sigma_{22}$ and $\sigma_{12}$ are shown in Figure 10. Due to the realignment of fibers towards the loading direction, the top face of the strip moves in the $-\mathbf{E}_{2}$ direction. The Cauchy stress component $\sigma_{11}$ in the upper left and lower right corners is larger than in the other regions, and that is similarly the case for the shear stress component $\sigma_{12}$. We emphasize that the lateral stress $\sigma_{22}$ is non-zero near the top and bottom faces but near the center of the strip it is almost zero, as can be seen in Figure 10(b). This allows us to verify the finite element analysis results with the assumption $\sigma_{22}=0$ for the specific element in the red frame shown in Figure 10(a). That is also the reason why we chose this particular element for the analytical calculation. As a result we obtained an exact match between the analytical solution and the FEAP computation.

\subsubsection{Simple Shear}

In the present example we test the capability of the proposed planar fiber dispersion model by subjecting a unit cube (hexahedral element) to a simple shear deformation. The bottom surface of the cube is fixed, and then we apply a horizontal displacement on the top surface, as shown in Figure 11. The mean fiber direction $\mathbf{M}$ is taken to be at an angle of $\Theta_{\mathrm{M}}$ relative to the $\mathbf{E}_{1}$ direction with values $0^{\circ}, 45^{\circ}$ or $90^{\circ}$. 


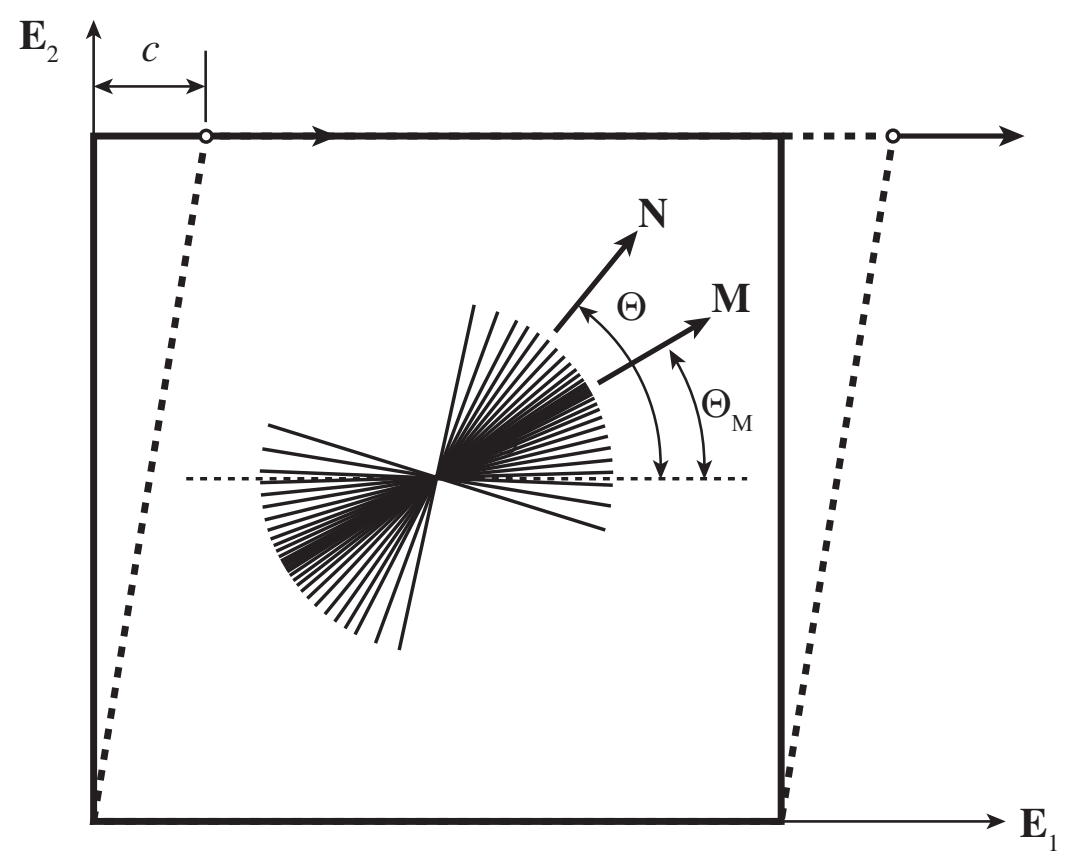

Figure 11: Simple shear of a unit cube in the $\left(\mathbf{E}_{1}, \mathbf{E}_{2}\right)$-plane. The mean fiber direction $\mathbf{M}$ in the reference configuration is shown with a symmetric dispersion and a general fiber direction $\mathbf{N} ; \Theta_{M}$ and $\Theta$ are the angles between $\mathbf{M}$ and $\mathbf{N}$ and the $\mathbf{E}_{1}$ direction, respectively. The dashed outline shows the deformed configuration, while $c$ denotes the amount of shear.

Thus, the deformation gradient and the right Cauchy-Green tensor in matrix form are given by

$$
[\mathbf{F}]=\left[\begin{array}{lll}
1 & c & 0 \\
0 & 1 & 0 \\
0 & 0 & 1
\end{array}\right], \quad[\mathbf{C}]=\left[\begin{array}{lcr}
1 & c & 0 \\
c\left(1+c^{2}\right) & 0 \\
0 & 0 & 1
\end{array}\right],
$$

where $c$ denotes the amount of shear. Hence, the components of $\mathbf{e}_{1}, \mathbf{e}_{2}, \mathbf{e}_{3}$ are

$$
\left[\mathbf{e}_{1}\right]=[\mathbf{F}]\left[\mathbf{E}_{1}\right]=[1,0,0], \quad\left[\mathbf{e}_{2}\right]=[\mathbf{F}]\left[\mathbf{E}_{2}\right]=[c, 1,0], \quad\left[\mathbf{e}_{3}\right]=[\mathbf{F}]\left[\mathbf{E}_{3}\right]=[0,0,1] .
$$

For an arbitrary in-plane fiber direction $\mathbf{N}(\Theta)=\cos \Theta \mathbf{E}_{1}+\sin \Theta \mathbf{E}_{2}$ the invariant $I_{4}$ is given by

$$
I_{4}=1+c \sin 2 \Theta+c^{2} \sin ^{2} \Theta
$$

From (69) the shear stress is

$$
\sigma_{12}=(\mu+\beta) c+\gamma
$$

and the normal stresses are

$$
\sigma_{11}=-p+\mu+\alpha+(\mu+\beta) c^{2}+2 \gamma c, \quad \sigma_{22}=-p+\mu+\beta
$$




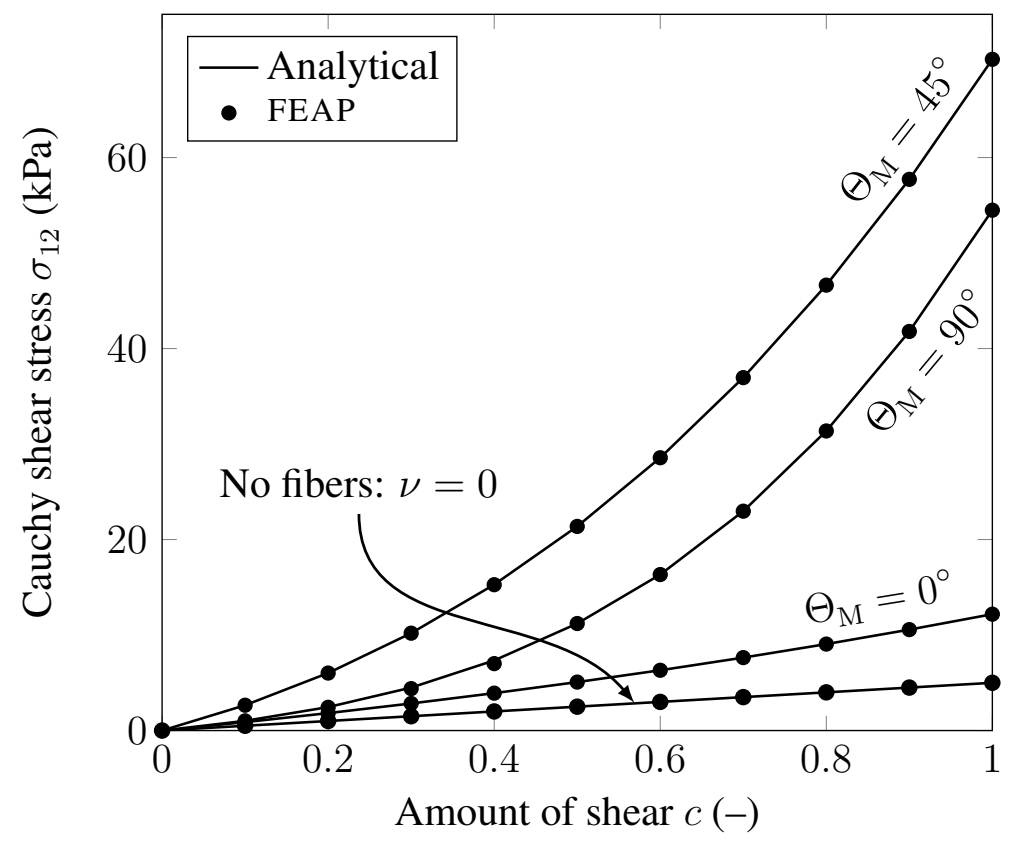

Figure 12: Comparison of the analytical expression (87) for the Cauchy shear stress $\sigma_{12}$ with the numerical results obtained from FEAP for the three mean fiber angles $\Theta_{\mathrm{M}}=0^{\circ}, 45^{\circ}$ and $90^{\circ}(\nu=5 \mathrm{kPa}, \mu=10 \mathrm{kPa}, b=2.9)$, and the corresponding results in the absence of fibers $(\nu=0)$.

Comparison between the analytical formulae (87) and the numerical results obtained from FEAP for three different mean fiber directions using the same material parameters as in Section 4.1.1 are shown in Figure 12.

To determine the Lagrange multiplier several options are possible. First, following [32], if the normal component of the surface traction on the inclined faces of the deformed cube is assumed to be zero, then

$$
\sigma_{11}+c^{2} \sigma_{22}-2 c \sigma_{12}=0
$$

Substituting (87) and (88) into (89) yields the Lagrange multiplier $p=(\alpha+\mu) /\left(1+c^{2}\right)$. Hence, we obtain the normal stresses

$$
\sigma_{11}=\mu\left(1+c^{2}\right)+\alpha+\beta c^{2}+2 \gamma c-\frac{\alpha+\mu}{1+c^{2}}, \quad \sigma_{22}=\mu+\beta-\frac{\alpha+\mu}{1+c^{2}} .
$$

A second possibility for determining $p$ is to set $\sigma_{33}=0$, which yields

$$
\sigma_{11}=\alpha+2 \gamma c+(\mu+\beta) c^{2}, \quad \sigma_{22}=\beta \text {. }
$$

Note that the shear stress $\sigma_{12}$ is independent of $p$. 


\subsection{D Fiber Dispersion Model}

Similarly to Section 4.1, we begin with a uniaxial extension test in the mean fiber direction of the specimen and then we present results for uniaxial extension in a direction other than the mean fiber direction. Finally we consider the simple shear deformation. But here, instead of a planar PDF (61), we consider the 3D fiber dispersion rotationally symmetric about the mean fiber direction according to (64). Now we can use (64) with $\mathbf{M} \cdot \mathbf{N}=\cos \vartheta$ to obtain

$$
\rho(\vartheta)=4 \sqrt{\frac{b}{2 \pi}} \frac{\exp \left(2 b \cos ^{2} \vartheta\right)}{\operatorname{erfi}(\sqrt{2 b})}
$$

where $\vartheta \in[0, \pi]$.

\subsubsection{Uniaxial Extension}

In this example, as in Section 4.1.1, we assume that the mean fiber direction $\mathbf{M}$ and the loading direction are aligned with $\mathbf{E}_{1}$. Because the dispersion is rotationally symmetric and the material is incompressible the deformation gradient and the Cauchy-Green tensors have matrices

$$
[\mathbf{F}]=\operatorname{diag}\left[\lambda, \lambda^{-1 / 2}, \lambda^{-1 / 2}\right], \quad[\mathbf{b}]=[\mathbf{C}]=\operatorname{diag}\left[\lambda^{2}, \lambda^{-1}, \lambda^{-1}\right],
$$

where $\lambda$ is the stretch in the $\mathbf{E}_{1}$ direction. For this special case the Cartesian basis vectors $\mathbf{E}_{i}=\mathbf{V}_{i}, i=1,2,3$, are the eigenvectors of $\mathbf{C}$. Then,

$$
\left[\mathbf{e}_{1}\right]=[\mathbf{F}]\left[\mathbf{E}_{1}\right]=[\lambda, 0,0], \quad\left[\mathbf{e}_{2}\right]=[\mathbf{F}]\left[\mathbf{E}_{2}\right]=\left[0, \lambda^{-1 / 2}, 0\right], \quad\left[\mathbf{e}_{3}\right]=[\mathbf{F}]\left[\mathbf{E}_{3}\right]=\left[0,0, \lambda^{-1 / 2}\right] .
$$

Given the decoupled form of the Cauchy stress tensor in (40) and the 3D isochoric Cauchy stress tensor (17) based on (40), the corresponding Cauchy stress tensor $\boldsymbol{\sigma}$ for an incompressible material is

$$
\begin{aligned}
\boldsymbol{\sigma}= & -p \mathbf{I}+\mu \mathbf{b}+\alpha \mathbf{e}_{3} \otimes \mathbf{e}_{3}+\beta \mathbf{e}_{1} \otimes \mathbf{e}_{1}+\beta^{\prime} \mathbf{e}_{2} \otimes \mathbf{e}_{2}+\gamma\left(\mathbf{e}_{3} \otimes \mathbf{e}_{1}+\mathbf{e}_{1} \otimes \mathbf{e}_{3}\right) \\
& +\gamma^{\prime}\left(\mathbf{e}_{3} \otimes \mathbf{e}_{2}+\mathbf{e}_{2} \otimes \mathbf{e}_{3}\right)+\delta\left(\mathbf{e}_{1} \otimes \mathbf{e}_{2}+\mathbf{e}_{2} \otimes \mathbf{e}_{1}\right),
\end{aligned}
$$

where we have adopted the neo-Hookean model for the matrix material, and where $\alpha, \beta, \beta^{\prime}, \gamma$, $\gamma^{\prime}$ and $\delta$ are given by (34)-(39) specialized to rotational symmetry. When we use the standard reinforcing model (5) for the collagen fibers, the coefficients can be simplified by using $\psi_{\mathrm{n}}^{\prime}=$ $\nu\left(I_{4}-1\right)$. For example, from (34), $\alpha$ gives

$$
\alpha=\nu \int_{0}^{\vartheta_{c}} \rho(\vartheta)\left(I_{4}(\vartheta)-1\right) \cos ^{2} \vartheta \sin \vartheta \mathrm{d} \vartheta
$$




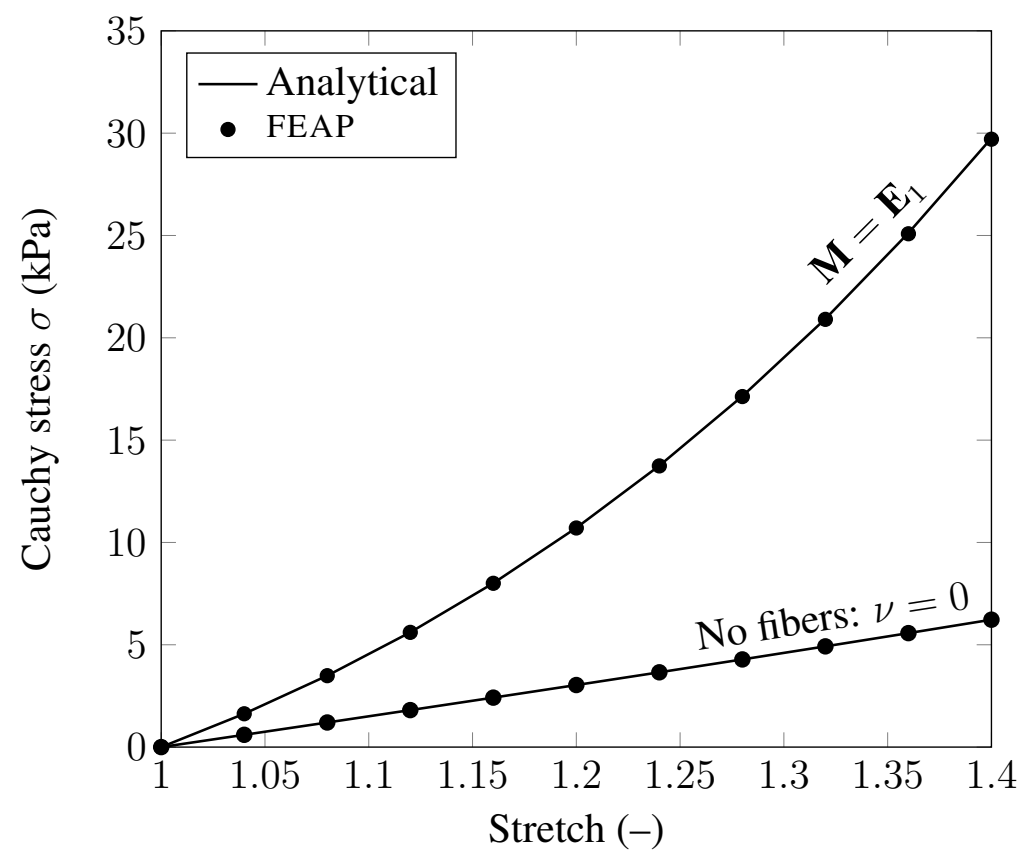

Figure 13: Comparison of the analytical solution obtained by using MATLAB and the FEAP computation for a uniaxial extension test in the mean fiber direction $\mathbf{M}=\mathbf{E}_{1}$ (and $\nu=10 \mathrm{kPa}$ ), together with the MATLAB results for the absence of fibers $(\nu=0) ; \mu=2 \mathrm{kPa}$ in each case.

where $I_{4}=\lambda^{2} \cos ^{2} \vartheta+\lambda^{-1} \sin ^{2} \vartheta$ and $\tan \vartheta_{\mathrm{c}}=\sqrt{\lambda(\lambda+1)}$ with $0<\vartheta_{\mathrm{c}}<\pi / 2$. Substituting (93) and (94) into (95), we obtain the Cauchy stress $\sigma$ in the $\mathbf{E}_{1}$ direction as

$$
\sigma=-p+(\mu+\beta) \lambda^{2}
$$

By using the boundary condition $\sigma_{22}=\sigma_{33}=0$, we find the Lagrange multiplier $p=(\mu+$ $\left.\beta^{\prime}\right) \lambda^{-1}$, and $\sigma$ becomes

$$
\sigma=(\mu+\beta) \lambda^{2}-\left(\mu+\beta^{\prime}\right) \lambda^{-1}
$$

Comparison between the FEAP computation and the analytical solution obtained with MATLAB is presented in Figure 13, with the material parameters $\mu=2 \mathrm{kPa}, \nu=10 \mathrm{kPa}$ and $b=2.9$, and compared with the result for $\nu=0$.

Similarly to Section 4.1.2, we have analyzed the uniaxial extension of a strip but with the general 3D fiber dispersion model and with mean fiber angles $\Theta_{\mathrm{M}}=90^{\circ}$ and $\Phi_{\mathrm{M}}=60^{\circ}$, where

$$
\mathbf{M}=\sin \Theta_{\mathrm{M}} \cos \Phi_{\mathrm{M}} \mathbf{E}_{1}+\sin \Theta_{\mathrm{M}} \sin \Phi_{\mathrm{M}} \mathbf{E}_{2}+\cos \Theta_{\mathrm{M}} \mathbf{E}_{3}
$$

Unlike the plane strain deformation considered in Section 4.1.2 the deformation is 3D so that there is a movement in the $\mathbf{E}_{3}$ direction of the front and back faces. With the same material 


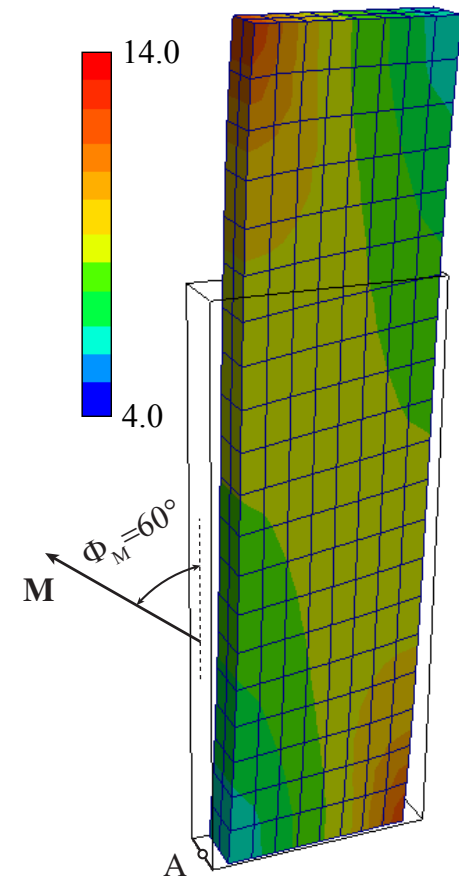

(a) $\sigma_{11}$

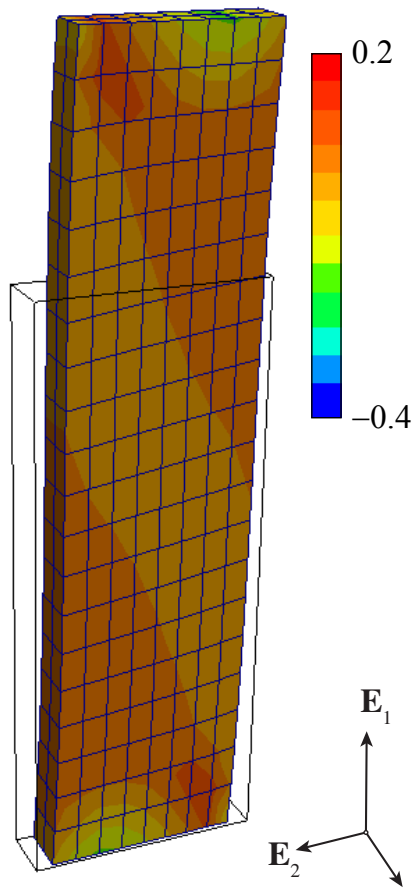

(b) $\sigma_{22}$

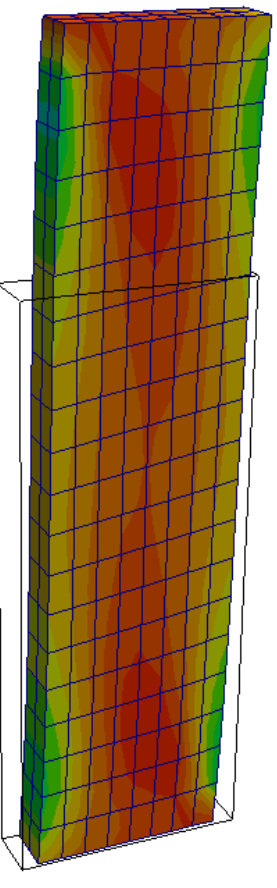

(c) $\sigma_{12}$

Figure 14: FEAP computation results for the uniaxial extension of a rectangular strip with the 3D fiber dispersion model and for the mean fiber direction $\mathbf{M}$ given by (99) with $\Theta_{M}=90^{\circ}$ and $\Phi_{\mathrm{M}}=60^{\circ}$. The Cauchy stress distributions (a), (b), (c) are plotted for an axial stretch of $\lambda=1.4$ with parameters $\mu=5 \mathrm{kPa}, \nu=10 \mathrm{kPa}$ and $b=2.9$.

parameters as in Section 4.1.2, we observe similar patterns for the Cauchy stress distributions in Figure 14 compared with those in Figure 10. However, the magnitudes of the stresses are much lower than for the planar case because the fiber density (92) is distributed in three dimensions rather than two, and without restriction to plane strain. Note, in particular, that there is a small contraction in the thickness direction.

\subsubsection{Simple Shear}

In this section, similarly to Section 4.1.3, we present results for a unit cube (hexahedral element) under simple shear but now with the rotationally symmetric 3D fiber dispersion model. We assume that the mean fiber direction is oriented at an angle $135^{\circ}$ from the $\mathbf{E}_{3}$ direction in the $(1,3)$-plane in the reference configuration, as illustrated in Figure 15. The bottom face of the cube in the $(1,2)$-plane is fixed, and we then apply a horizontal displacement on the top surface, as shown in Figure 15. The deformation gradient and the right Cauchy-Green tensor have the 


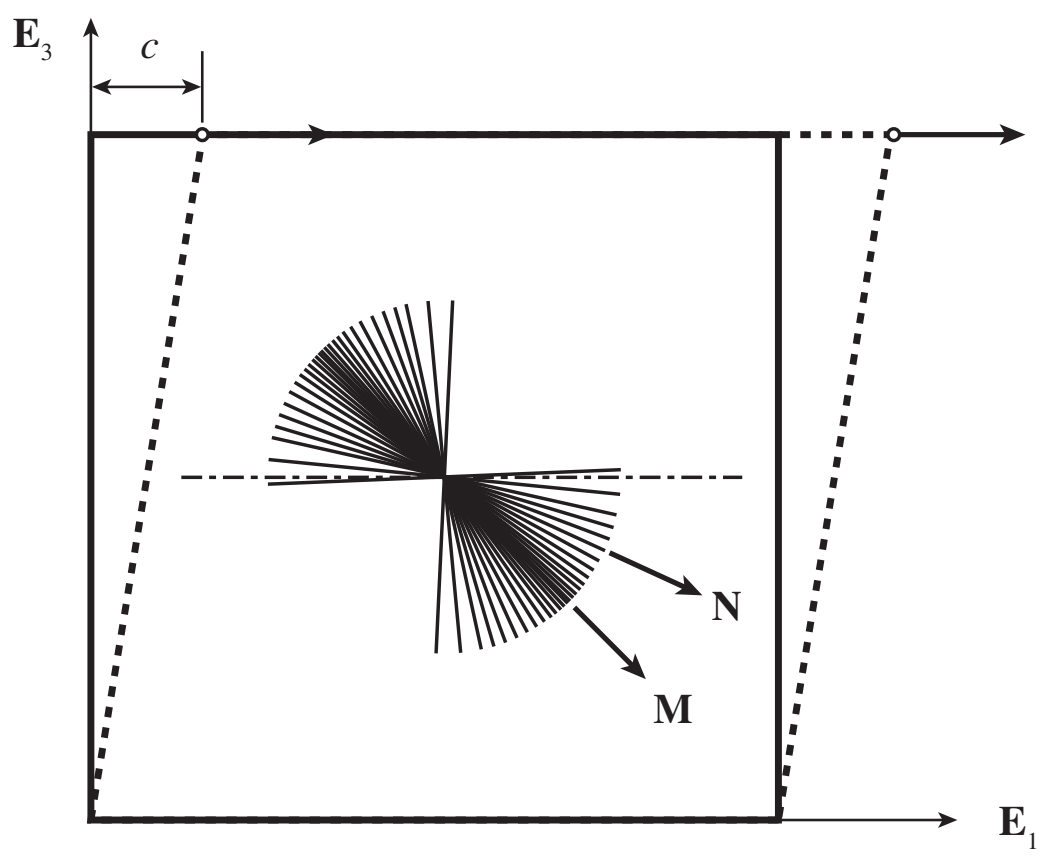

Figure 15: Simple shear of a unit cube in the $\left(\mathbf{E}_{1}, \mathbf{E}_{3}\right)$-plane. The mean fiber direction $\mathbf{M}$ in the reference configuration lies in the $\left(\mathbf{E}_{1}, \mathbf{E}_{3}\right)$-plane. The fiber dispersion is rotationally symmetric about $\mathbf{M}$, and $\mathbf{N}$ is a general fiber direction in this dispersion. The dashed outline shows the deformed configuration, where $c$ is the amount of shear.

matrix representations

$$
[\mathbf{F}]=\left[\begin{array}{lll}
1 & 0 & c \\
0 & 1 & 0 \\
0 & 0 & 1
\end{array}\right], \quad[\mathbf{C}]=\left[\begin{array}{ccc}
1 & 0 & c \\
0 & 1 & 0 \\
c & 0 & \left(1+c^{2}\right)
\end{array}\right] .
$$

Thus, the vectors $\mathbf{e}_{i}=\mathbf{F E}_{i}, i=1,2,3$, have components

$$
\left[\mathbf{e}_{1}\right]=[\mathbf{F}]\left[\mathbf{E}_{1}\right]=[1,0,0], \quad\left[\mathbf{e}_{2}\right]=[\mathbf{F}]\left[\mathbf{E}_{2}\right]=[0,0,1], \quad\left[\mathbf{e}_{3}\right]=[\mathbf{F}]\left[\mathbf{E}_{3}\right]=[c, 0,1] .
$$

Instead of using eigenvectors of $\mathbf{C}$, as in (27), for this particular example we represent $\mathbf{N}$ with respect to the Cartesian basis vectors $\mathbf{E}_{i}, i=1,2,3$ :

$$
\mathbf{N}=\sin \Theta \cos \Phi \mathbf{E}_{1}+\sin \Theta \sin \Phi \mathbf{E}_{2}+\cos \Theta \mathbf{E}_{3} .
$$

Then $I_{4}(\mathbf{N})=\mathbf{C}: \mathbf{N} \otimes \mathbf{N}$ reads

$$
I_{4}=1+c^{2} \cos ^{2} \Theta+c \sin 2 \Theta \cos \Phi .
$$

Because $c$ is always positive in this example, the integration domain $\Omega$ is now defined by

$$
c \cos ^{2} \Theta+\sin 2 \Theta \cos \Phi>0,
$$




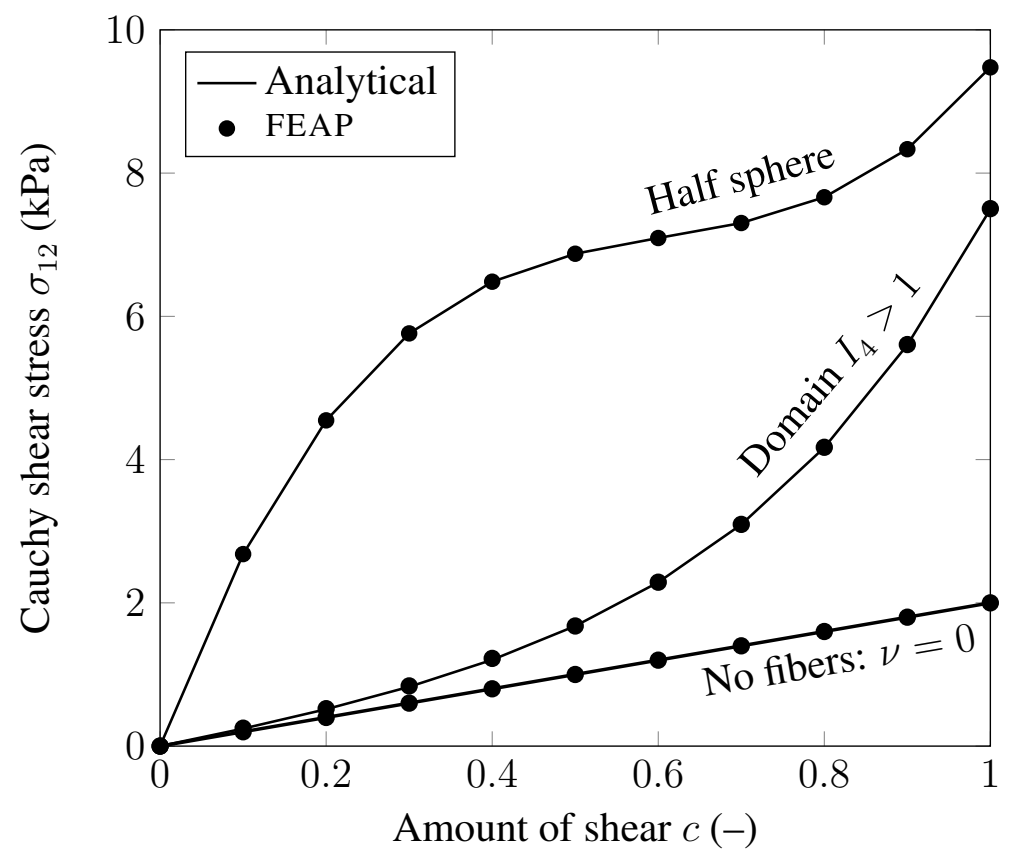

Figure 16: Comparison of the predictions of FEAP and the analytical solutions based on (105) and MATLAB with $\alpha$ and $\gamma$ determined by integration over the relevant domain, which is either $I_{4}>1$ or the half sphere $(\mu=2 \mathrm{kPa}, \nu=50 \mathrm{kPa})$.

for $\Theta \in[0, \pi], \Phi \in[-\pi / 2, \pi / 2]$. Based on the general form of the Cauchy stress tensor (95), the shear stress in the $(1,2)$-plane is

$$
\sigma_{12}=(\mu+\alpha) c+\gamma
$$

The normal stresses can be determined by the same method as in Section 4.1.3, but note that in this case the shear stresses $\sigma_{13}$ and $\sigma_{23}$ are non-zero. The coefficients $\alpha$ and $\gamma$ in (105), which depend on $c$, should be integrated in the domain defined by (104), which may be achieved by using the Boole operation and the NIntegrate function in MATHEMATICA (Wolfram Research, Champaign, IL, USA). Comparison between the FEAP computation and the analytical solution obtained by using (105) and MATHEMATICA with the material parameters $\mu=2 \mathrm{kPa}$, $\nu=50 \mathrm{kPa}$ and $b=2.9$ is presented in Figure 16. Note that in the FEAP implementation the formulation presented in Section 2.3 with $\mathbf{N}$ described in terms of the eigenvectors of $\mathbf{C}$ is used. In Figure 16 we have also shown the analytical and FEAP simulation results for the Cauchy shear stress $\sigma_{12}$ versus the amount of shear $c$ for the case in which the coefficients $\alpha$ and $\gamma$ in (105) are obtained by integration over the half sphere defined by $\Theta \in[0, \pi], \Phi \in[-\pi / 2, \pi / 2]$. As shown, the difference between the shear stresses from the two methods increases with the amount of shear but then gradually reduces as the shear increases. This can be explained by 
the fact that as the shear increases more fibers becomes stretched and fewer fibers are excluded from the integration domain.

Note that if $\mathbf{N}=\mathbf{M}$, with $\Phi=0$ and $\Theta=135^{\circ}$, then $I_{4}(\mathbf{M})=1-c+c^{2} / 2$ from (103) which is always less than one for any $c \in(0,1)$. Thus, with the original model in [11] no fiber contribution would be considered and the material would be simply neo-Hookean. However, with the current model the fiber contribution is accounted for even if the stretch in the mean fiber direction is always less than one.

\section{Concluding Remarks}

In the paper [15] we have introduced a dispersed fiber model expressed as a weighted strainenergy function that allows the contribution of fibers that are shortened to be excluded from the energy function. The model was developed for planar and for general three-dimensional deformations. This model uses the angular integration (AI) approach in contrast to a generalized structure tensor (GST) approach. It identifies theoretically the boundary of the integration domain for which the fibers are extended. In the present paper we focus on the computational implementation of the dispersion model proposed in [15]. In particular, the analytical expression of the elasticity tensor and the integration boundary in the deformation space within which fibers are extended is provided. We have proposed an adaptive finite element integration scheme which allows the stress and the elasticity tensors to be obtained numerically by integration over the appropriate domain. We have illustrated the computational method with several examples, indicating the efficacy of the dispersion model. In addition, we have shown that the exclusion of the compressed fibers makes a significant difference to the elastic response compared with the situation where they are not excluded. This method is suitable for solving more general boundary-value problems, and it can also be applied within the framework of the GST approach, work on which is in progress and the results of which will be reported separately.

\section{References}

[1] P. B. Canham, H. M. Finlay, J. G. Dixon, D. R. Boughner, and A. Chen. Measurements from light and polarised light microscopy of human coronary arteries fixed at distending pressure. Cardiovasc. Res., 23:973-982, 1989.

[2] H. M. Finlay, L. McCullough, and P. B. Canham. Three-dimensional collagen organization of human brain arteries at different transmural pressures. J. Vasc. Res., 32:301-312, 1995. 
[3] H. M. Finlay, P. Whittaker, and P. B. Canham. Collagen organization in the branching region of human brain arteries. Stroke, 29:1595-1601, 1998.

[4] A. J. Schriefl, G. Zeindlinger, D. M. Pierce, P. Regitnig, and G. A. Holzapfel. Determination of the layer-specific distributed collagen fiber orientations in human thoracic and abdominal aortas and common iliac arteries. J. R. Soc. Interface, 9:1275-1286, 2012.

[5] A. J. Schriefl, H. Wolinski, P. Regitnig, S. D. Kohlwein, and G. A. Holzapfel. An automated approach for 3D quantification of fibrillar structures in optically cleared soft biological tissues. J. R. Soc. Interface, 10:20120760, 2013.

[6] W. J. Karlon, J. W. Covell, A. D. McCulloch, J. J. Hunter, and J. H. Omens. Automated measurement of myofiber disarray in transgenic mice with ventricular expression of ras. Anat. Rec., 252:612-625, 1998.

[7] J. W. Covell. Tissue structure and ventricular wall mechanics. Circulation, 118:699-701, 2008.

[8] C. Boote, S. Dennis, and K. Meek. Spatial mapping of collagen fibril organisation in primate cornea-an X-ray diffraction investigation. J. Struct. Biol., 146:359-367, 2004.

[9] C. Boote, S. Dennis, Y. Huang, A. J. Quantock, and K. M. Meek. Lamellar orientation in human cornea in relation to mechanical properties. J. Struct. Biol., 149:1-6, 2005.

[10] M. B. Lilledahl, D. M. Pierce, T. Ricken, G. A. Holzapfel, and C. de Lange Davies. Structural analysis of articular cartilage using multiphoton microscopy: input for biomechanical modeling. IEEE Trans. Med. Imaging, 30:1635-1648, 2011.

[11] T. C. Gasser, R. W. Ogden, and G. A. Holzapfel. Hyperelastic modelling of arterial layers with distributed collagen fibre orientations. J. R. Soc. Interface, 3:15-35, 2006.

[12] D. H. Cortes, S. P. Lake, J. A. Kadlowec, L. J. Soslowsky, and D. M. Elliot. Characterizing the mechanical contribution of fiber angular distribution in connective tissue: comparison of two modeling approaches. Biomech. Model. Mechanobiol., 9:651-658, 2010.

[13] Y. Lanir. Constitutive equations for fibrous connective tissues. J. Biomech., 16:1-12, 1983.

[14] G. A. Holzapfel, J. A. Niestrawska, R. W. Ogden, A. J. Reinisch, and A. J. Schriefl. Modelling non-symmetric collagen fibre dispersion in arterial walls. J. R. Soc. Interface, 12:20150188, 2015. 
[15] G. A. Holzapfel and R. W. Ogden. On the tension-compression switch in soft fibrous solids. Eur. J. Mech. A/Solids, 49:561-569, 2015.

[16] Abaqus 6.13 Analysis User's Guide. Section 22.5.3: Anisotropic hyperelastic behavior, 2013.

[17] G. A. Ateshian, B. J. Ellis, and J. A. Weiss. Equivalence between short-time biphasic and incompressible elastic material responses. J. Biomech. Eng., 129:405-412, 2007.

[18] G. A. Ateshian, V. Rajan, N. O. Chahine, C. E. Canal, and C. T. Hung. Modeling the matrix of articular cartilage using a continuous fiber angular distribution predicts many observed phenomena. J. Biomech. Eng., 131:61003, 2009.

[19] S. Federico and T. C. Gasser. Nonlinear elasticity of biological tissues with statistical fibre orientation. J. R. Soc. Interface, 7:955-966, 2010.

[20] A. V. Melnik, H. Borja Da Rocha, and A. Goriely. On the modeling of fiber dispersion in fiber-reinforced elastic materials. Int. J. Non-Linear Mech., 75:92-106, 2015.

[21] P. J. Flory. Thermodynamic relations for highly elastic materials. Trans. Faraday Soc., 57:829-838, 1961.

[22] R. W. Ogden. Nearly isochoric elastic deformations: Application to rubberlike solids. J. Mech. Phys. Solids, 26:37-57, 1978.

[23] G. A. Holzapfel. Nonlinear Solid Mechanics. A Continuum Approach for Engineering. John Wiley \& Sons, Chichester, 2000.

[24] G. A. Holzapfel, T. C. Gasser, and R. W. Ogden. A new constitutive framework for arterial wall mechanics and a comparative study of material models. J. Elasticity, 61:1-48, 2000.

[25] G. Y. Qiu and T. J. Pence. Remarks on the behavior of simple directionally reinforced incompressible nonlinearly elastic solids. J. Elasticity, 49:1-30, 1997.

[26] H. Miyazaki and K. Hayashi. Tensile tests of collagen fibers obtained from the rabbit patellar tendon. Biomed. Microdevices, 2:151-157, 1999.

[27] FEAP - A Finite Element Analysis Program, Version 8.2 User Manual. University of California at Berkeley, Berkeley, California, 2008. 
[28] J. Berntsen, T. O. Espelid, and A. Genz. An adaptive algorithm for the approximate calculation of multiple integrals. ACM Trans. Math. Software, 17:437-451, 1991.

[29] J. Berntsen, T. O. Espelid, and A. Genz. An adaptive multidimensional integration routine for a vector of integrals. ACM Trans. Math. Software, 17:452-456, 1991.

[30] H. Xiao and Z. Gimbutas. A numerical algorithm for the construction of efficient quadrature rules in two and higher dimensions. Comput. Math. Appl., 59:663-676, 2010.

[31] J. C. Simo and R. L. Taylor. Quasi-incompressible finite elasticity in principal stretches. Continuum basis and numerical algorithms. Comput. Meth. Appl. Mech. Eng., 85:273$310,1991$.

[32] C. O. Horgan and J. G. Murphy. On the normal stresses in simple shearing of fiberreinforced nonlinearly elastic materials. J. Elasticity, 104:343-355, 2011. 